\title{
At the source of the polarisation of auroral emissions: experiments and modeling
} \author{
Alain Delboulbé ${ }^{1}$, Stéphane Curaba ${ }^{1}$, and Magnar Gullikstad Johnsen ${ }^{4}$ (E) \\ ${ }^{1}$ Institut de Planétologie et d'Astrophysique de Grenoble (IPAG) CNRS UGA, 3800 Grenoble, France \\ ${ }^{2}$ Univ. Grenoble Alpes, Univ. Savoie Mont Blanc, CNRS, IRD, UGE, ISTerre, 38000 Grenoble, France \\ ${ }^{3}$ Univ. Lille, CNRS, UMR 8518 - LOA Laboratoire d'Optique Atmosphérique, 59000 Lille, France \\ ${ }^{4}$ Troms $\varnothing$ Geophysical Observatory, UiT - The Arctic University of Norway, 9019 Troms $\varnothing$, Norway \\ ${ }^{5}$ Honorary Astronomer at Royal Observatory of Belgium, Avenue Circulaire 3, 1180 Brussels, Belgium
}

Léo Bosse $^{1}$ (D), Jean Lilensten ${ }^{1,5}{ }^{*}$, Nicolas Gillet ${ }^{2}$ (1), Colette Brogniez ${ }^{3}$, Olivier Pujol ${ }^{3}$, Sylvain Rochat ${ }^{1}$,

Received 29 July 2021 / Accepted 17 February 2022

\begin{abstract}
A polarised radiative transfer model (POMEROL) has been developed to compute the polarisation measured by a virtual instrument in a given nocturnal environment. This single-scattering model recreates real-world conditions (among them atmospheric and aerosol profiles, light sources with complex geometries at the ground and in the sky, terrain obstructions). It has been successfully tested at mid-latitudes where sky emissions are of weak intensity. We show a series of comparisons between POMEROL predictions and polarisation measurements during two field campaigns in the auroral zone, in both quiet and active conditions. These comparisons show the strength of the model to assess the aerosol characteristics in the lower atmosphere by using a mesospheric line. They also show that three main upper atmosphere emissions must be polarised: the green atomic oxygen line at $557.7 \mathrm{~nm}$ and the $1^{\text {st }} \mathrm{N}_{2}^{+}$negative band at $391.4 \mathrm{~nm}$ (purple) and $427.8 \mathrm{~nm}$ (blue). This polarisation can be either created directly at the radiative de-excitation or may occur when the non-polarised emission crosses the ionospheric currents. We provide some of the potentialities it offers in the frame of space weather. These require refinements of the preliminary modeling approach considered in the present study.
\end{abstract}

Keywords: Auroral lights / Linear polarisation / Radiative transfer / Ionospheric environment

\section{Introduction}

Since the first half of the 20th century, several attempts were made to observe the polarisation properties of upper atmospheric emissions, such as aurorae or nightglow (Harang, 1933; Duncan, 1959). However, the lack of an accepted physical model and the variety of measurements could not deliver a definite answer (Chamberlain, 1959; Harang, 1960). Recently, Lilensten et al. (2006) have dug up the problem: are the upper atmospheric emissions polarised? And if so, can we measure and use it to draw conclusions on the processes at play at the emission?

Indeed, the Earth's upper atmosphere forms a dynamical frontier between space and ground. It is at the origin of observational difficulties for the study of the Earth's internal magnetic field (Finlay et al., 2017). A better understanding and modeling of the upper atmosphere could also improve space weather forecasting (Akmaev, 2011). A remaining issue is the inaccessibility of the upper atmosphere, where balloons and rockets can barely fly high enough, while Low Earth Orbits (LEOs) are still too high for in-situ measurements. Some indirect methods of observation exist, such as radars (Appleton, 1924; Lehtinen et al., 2002; Chisham et al., 2007), but they demand huge and costly instrumentation. Monitoring the upper atmosphere dynamics from ground polarisation measurements could improve the spatial and time coverage of observational constraints, as well as reduce the costs of the required technologies.

We recently developed a radiative transfer code designed to examine the nightglow polarisation (Bosse et al., 2021). This single-scattering model incorporates the contributions of light pollution from the ground, as well as the background sky glow (stars and airglow). It takes into account mechanisms such as Mie scattering by aerosols, Rayleigh scattering by air molecules, and possibly a polarisation at the emission. This model was successfully tested at mid-latitudes thanks to polarisation measurements at different wavelengths. It could reproduce observations in a light-polluted environment using only the

*Corresponding author: jean.lilensten@univ-grenoble-alpes.fr 


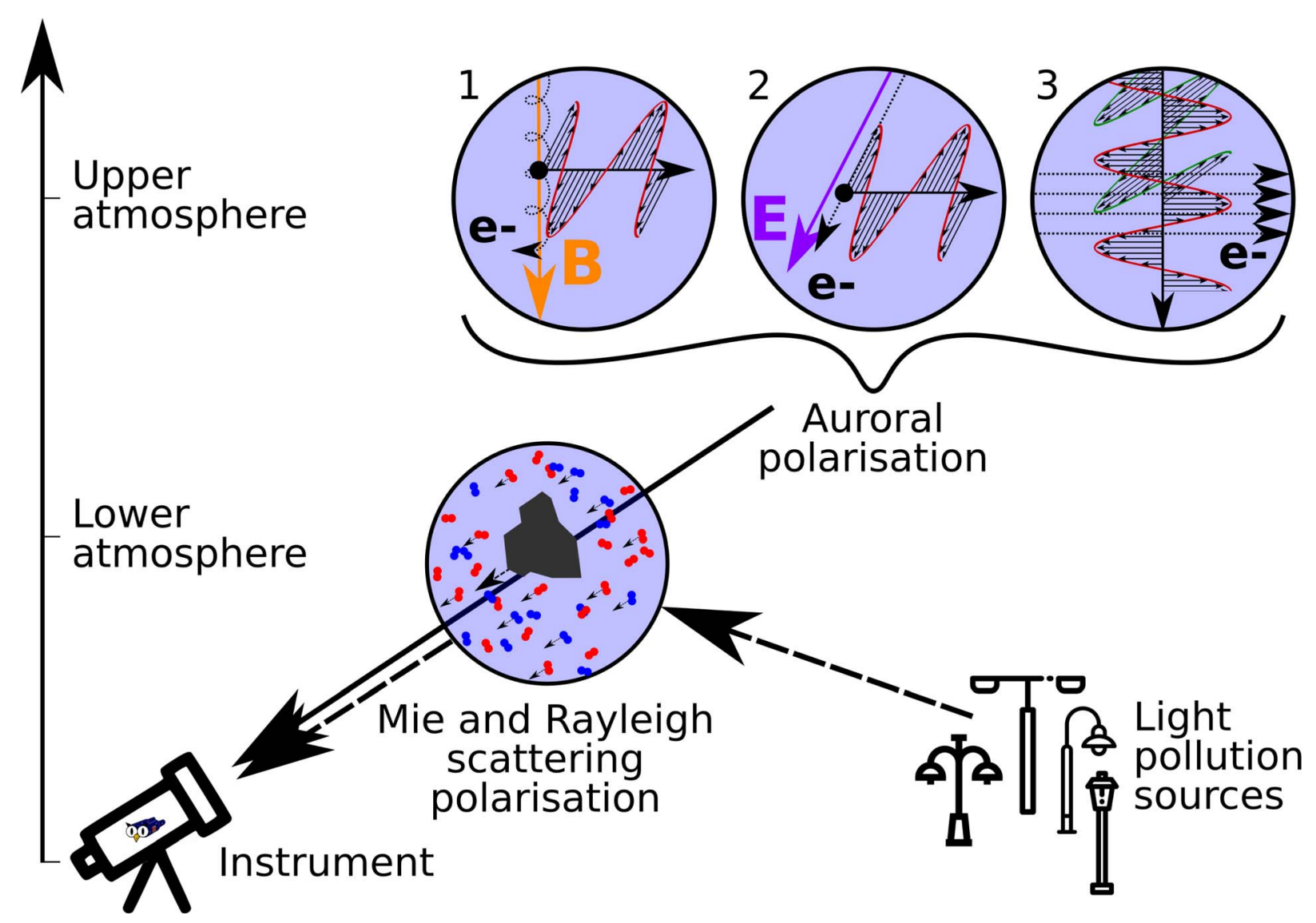

Fig. 1. Representation of the main physical processes at play in the following article. The instrument (lower left corner) observes two main light sources: the aurora (solid black arrows) and the scattering of another source of light in the lower atmosphere (dashed black arrows). The latter is represented by the center circle representing Mie and Rayleigh scattering on aerosol and air molecules. Its origin is shown here as street lamps but could be due to other sources like snow reflection or aurorae. The auroral polarisation model is uncertain at this point and could follow any of the three models represented in the top right numbered 1, 2, and 3. In those, the electron's motion direction is the thin dotted lines, in 1 , the magnetic field is the orange line (B), and in 2, the electric field is the purple line (E). Auroral emission is the thin black line with a polarisation direction represented by the red and green waves.

surrounding emissions map, an aerosol profile, and a constant and uniform skylight contribution.

Bosse et al. (2020) have conducted a series of experiments at high latitude to measure the polarisation of auroral lights as seen from the ground in the green $(557.7 \mathrm{~nm})$, blue $(427.8 \mathrm{~nm})$, and purple $(391.4 \mathrm{~nm})$ lines. They show that part of the polarisation is undoubtedly acquired along the line of sight through Rayleigh and/or Mie scattering. However, the complexity of their records also suggests some possible polarisation of auroral emissions already in the ionosphere. This paper aims to assess this possibility by modeling several polarisation sources (see Fig. 1): in the upper atmosphere or through scattering in the lower atmosphere. The latter may be due either to pollution sources on ground, or to indirect illumination of the instrument by auroral emissions all over the sky, or finally to the reflection of the aurorae on the snowy landscape. Compared with the polarisation induced by Mie and Rayleigh scattering, our understanding of the processes at play for the one originating at the emission of auroral lights is still in its infancy and requires heavy investigation. We envision three possible physical processes that may cause an auroral polarisation in the upper atmosphere. First, directly at the emission, as the impacting electrons can follow a magnetic or an electric field (circles 1 and 2 of Fig. 1). Then, in both cases, the polarisation direction can be either perpendicular (circle 1) or parallel (circle 2) to the incoming electron direction. The third hypothesis (circle 3 ) is a polarisation occurring while the light crosses a current. This current would act as a polarising filter that would absorb or deflect a specific polarisation direction preferentially. Note that these processes are not mutually exclusive.

The manuscript is organized as follows. In Section 2, we recall the main characteristics of the polarimeter, of the POMEROL model, and our strategy to account for the several sources of light. Next, we describe in Section 3 our measurements obtained in the auroral zone, and Section 4 their interpretation as seen through the POMEROL model. Finally, we discuss in Section 5 the geophysical implications of our results.

\section{Description of the instrument and of the POMEROL model}

A complete description of the POMEROL model (standing for "POlarisation par Mie Et Rayleigh des Objets Lumineux") is 
given in Bosse et al. (2021) for midlatitude applications where sky emissions (nightglow, skylight) are faint and mainly isotropic. This approximation does not hold anymore at high latitudes where aurorae are bright and show a complex geometry. We complete here the description of the model to take into account these environmental changes.

\subsection{Short description of the instrument}

The polarimeter used in this study has been fully described in Bosse et al. (2020). Therefore we only recall its basics. The auroral light is filtered through a polarising lens rotating at $2 \mathrm{~Hz}$. Immediately after, it passes through a narrow $10 \mathrm{~nm}$ width optical filter centered around the observed wavelength. The light then enters a photomultiplier with a sampling frequency of $1 \mathrm{kHz}$. A lock-in analysis is performed in real-time. The measured Degree of Linear Polarisation (DoLP) and Angle of Linear Polarisation (AoLP) are calibrated however, the flux is only given in arbitrary units. In the end, there are two data points per second, which are then smoothed using a sliding window of $10 \mathrm{~s}$ to reduce the error bars. In the following figures, we only show the averaged data for clarity.

\subsection{Main characteristics of the model}

The model takes as input several parameters: the virtual instrument setup (location, orientation, wavelength, aperture angle), the atmospheric model (vertical profiles for the air pressure, temperature, and aerosol content), and the emissions by light sources that surround the instrument. For a complete description of each of these inputs, see Bosse et al. (2021). Surrounding emission maps are deduced from broad-band satellite images of Earth taken during night-time and post-processed so as to remove ephemeral lights (Mills et al., 2013; Miller et al., 2013). Each pixel of these images is treated as an isotropic point source. Originally, the pixels were $46 \mathrm{~m}$ wide, but this resolution is downgraded to reduce unnecessary computation time. In this study, pixel sizes vary between $100 \mathrm{~m} \times 20 \mathrm{~m}$ close to the instrument to $7 \mathrm{~km} \times 20 \mathrm{~km}$ on the map border (100 km away from the instrument). These measures correspond to a polar grid with 31 radial and 31 azimutal bins. The radial length of each pixel is defined to increase with its distance to the instrument. The altitude of the terrain around the instrument can also be specified in order to compute the occultation by surrounding mountains. Given a set of entries for the instrumental setup, the atmospheric properties, and emissions maps, POMEROL integrates the contributions of all point sources along the line of sight to compute the flux, the DoLP and the AoLP of the light measured by the virtual instrument. An isotropic and unpolarised sky emission can be added to account for nightglow and starlight.

Note that multiple scattering in the atmosphere is not taken into account in the running version of POMEROL. A full discussion on the main impact expected when including multiple (with respect to single) scattering is provided in Bosse et al. (2021) and summarized hereafter. Overall, we can expect multiple scattering to decrease the modeled DoLP by a factor of 0.8 in the short wavelengths (blue and purple lines) compared to the single scattering and increase the flux by less than $20 \%$. However, we expect multiple scattering and scattering in the atmosphere as a whole to play a relatively weaker role in auroral conditions. Indeed in such a configuration, the proportion of scattered light should be relatively smaller than at mid-latitudes because brighter emissions will directly hit the instrument. Consequently, if lights emitted by aurorae are unpolarised, then the DoLP measured by the instrument (due to scattered light) should be lower. On the contrary, if lights emitted by aurorae carry some polarisation (as discussed below), this polarisation should be relatively less affected by (single or multiple) scattering.

Figure 2 shows a flowchart of the full POMEROL model. It includes additional inputs (in comparison with Bosse et al., 2021) necessary in the auroral conditions. These are described thereafter. From top to bottom, it represents the environmental inputs (in blue), the emission maps (pink), the computation of the ground and sky contributions (in green) via the radiative transfer code described in Bosse et al. (2021), and finally, the final model made of the linear combination of the previous contributions (see Sect. 2.4).

\subsection{Additional inputs at high latitudes}

\subsubsection{All sky camera images}

In order to account for the complex geometries of aurorae, the model considers as input all-sky camera images from the University of Oslo All-Sky Imager located at the Observatory (available here: http://tid.uio.no/plasma/aurora/data.php). The images are calibrated in radiance and are available at two wavelengths, $557.7 \mathrm{~nm}$ and $630.0 \mathrm{~nm}$. A calibration file gives the position (in azimuth and elevation) of each pixel in the sky. Several images are available per minute. Skylights from all directions indirectly hit the polarimeter via scattering in the atmosphere. The model can take a list of images as input in order to compute the evolution of the light properties as seen by the instrument. The raw data having a high resolution, the model predictions may require significant computation time. It may be reduced as the user can control the number of pixels used in the input maps. In this work, the image is downgraded to a thousand pixels. To test the model, several synthetic emissions can be considered. One may replace all-sky pictures with more simple configurations such as a point source emission, an isotropic emission, or more complex shapes such as controlled auroral arcs.

\subsubsection{Impact of the albedo}

Since the current observations occur in winter at high latitudes, the ground is covered with snow. For fresh snow, the albedo can reach values as high as 0.9 in the visible range so that the re-emission of auroral lights on the ground potentially constitutes a significant source of light. Because of the geographical properties of the terrain with clear limits between the forest and the meadow, the ground albedo primarily depends on the altitude. In general, the albedo is small for the sea and forest at low altitudes and increases when trees get scarcer. The model uses the sky emission map (from all-sky cameras) to compute its reflection on the ground. For sources " $s$ " of radiance $L_{s}$ in $\mathrm{nW} / \mathrm{m}^{2} / \mathrm{sr}$ and area $A_{\mathrm{s}}$ in the sky, a point on the ground with albedo $a$ re-emits over the upper half sphere with radiance

$$
L=\frac{a}{2 \pi} \sum_{s} \frac{L_{s} A_{s}}{D_{s}^{2}} \sin \theta_{s},
$$




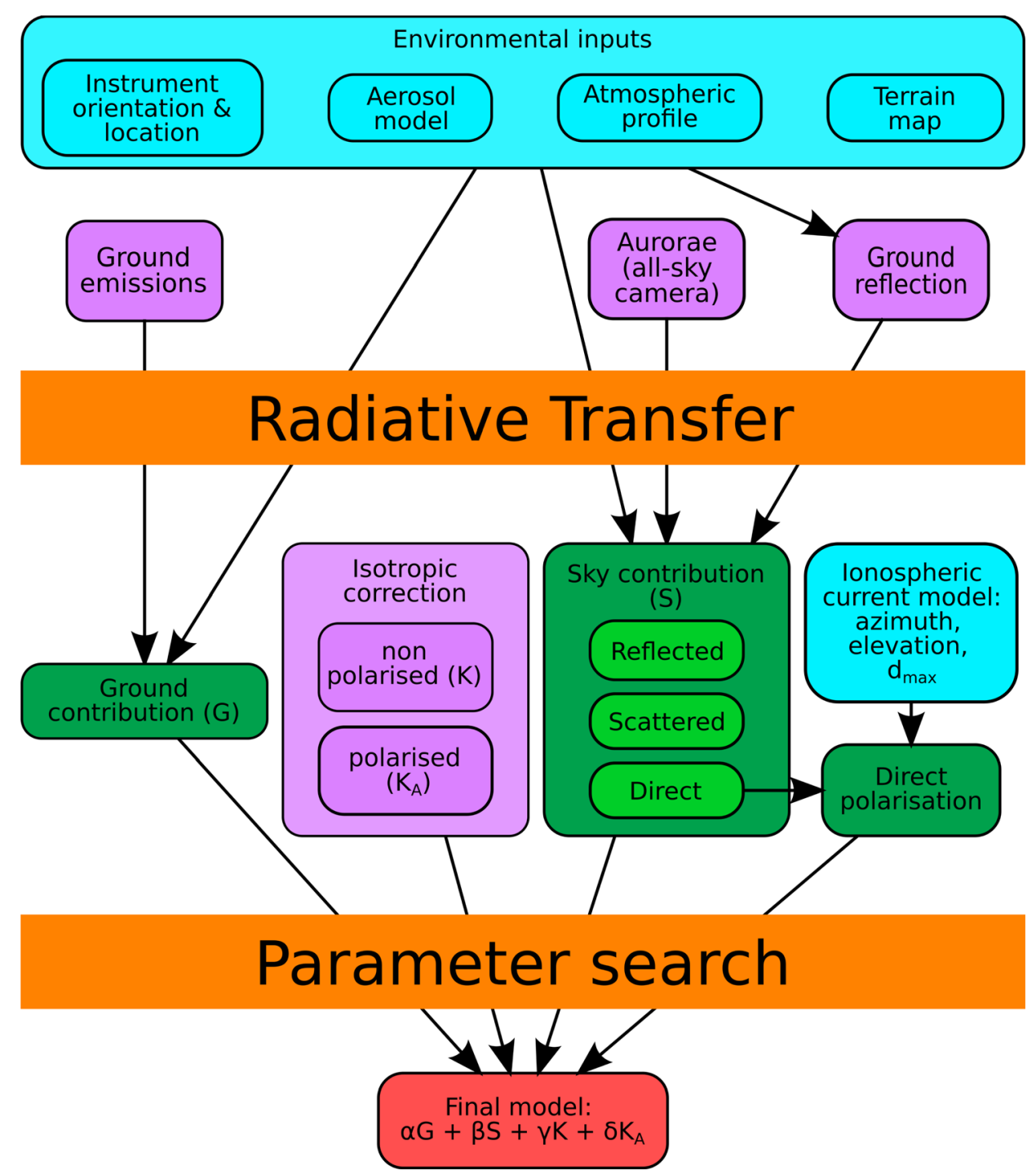

Fig. 2. POMEROL flowchart. Blue boxes: additional environmental inputs. Pink boxes: input emissions. Green boxes: Ground $(G)$ and sky $(S)$ models. Orange bands: main steps of the process. Red box: Final outputs in the same format as the measurements.

where $D_{\mathrm{s}}$ is the distance between the emission and the reflection point on the ground, and $\theta_{\mathrm{s}}$ is the elevation angle of the source as seen from the ground. The reflection on the ground is considered lambertian, hence the $2 \pi$ normalization. The radiance of each pixel of the ground albedo map is computed and is considered as an additional isotropic point source.

We study specifically the case of the reflection of the nightglow on the ground, potentially important on snowy surfaces. Considering the reflection on the snow to be polarised with a DoLP of the order of a few percent (Lv \& Sun, 2014) could in principle be achieved, but increases the complexity of the model tremendously for minimal effects on the main results obtained here. Thus, it is not implemented for now. However, the reflection on the ground then acquires polarisation from Rayleigh scattering by the air molecules and Mie scattering by aerosols.

The ground albedos are taken from Henderson-Sellers \& Wilson (1983). The typical average values are 0.8 for the snow 0.2 for a forest. We perform a simulation in the case of a virtual instrument surrounded by mountains corresponding to the Skibotn landscape (Norway), where the real experiments will be conducted (see Sect. 3 and the map in Appendix A). We study three different albedo profiles shown in Figure 3.

In the case of a uniform sky, the effect of the ground albedo on the measured DoLP is negligible (smaller than $0.3 \%$, not shown). However, in the presence of an aurora, when the emissions in the sky become very bright and anisotropic, one cannot rule out a significant impact of ground re-emissions. We, therefore, simulate an unpolarised auroral arc (see Fig. 4, top) as input for the model. This arc expands in the EastWest direction at $45^{\circ}$ elevation, Northward. Its relative intensity is 10 times brighter than the dark surrounding sky (respectively 100 and $10 \mathrm{nW} / \mathrm{m}^{2} / \mathrm{sr}$ ). We perform an almucantar $\left(360^{\circ}\right.$ rotation of the virtual instrument at a given elevation) at an elevation of $45^{\circ}$.

The flux, DoLP, and AoLP as seen by the virtual instrument are shown in Figure 4. The direct light (i.e., not scattered in the atmosphere) is the main contributor to the flux measured by the 


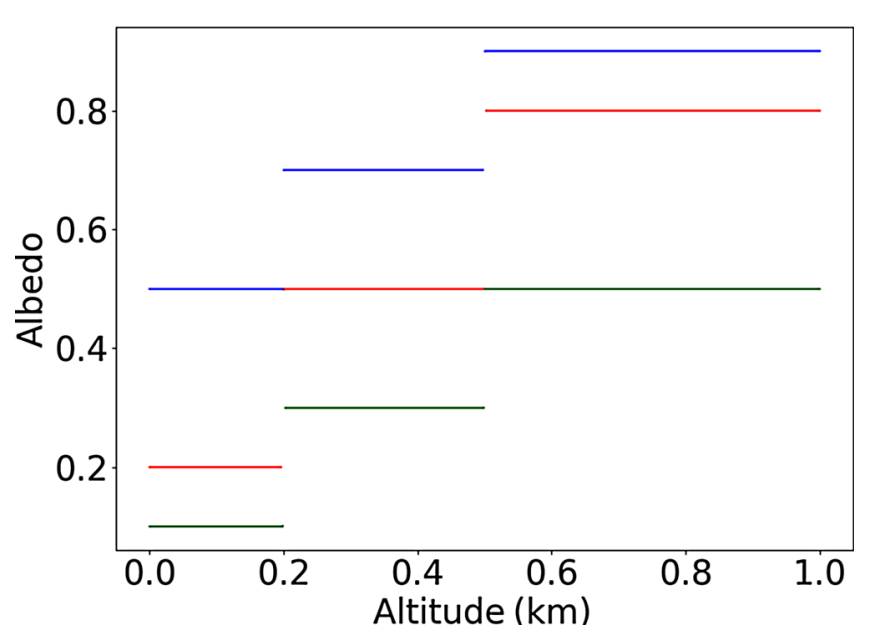

Fig. 3. Albedo profiles with altitude used throughout this study. The values increase with the altitude to match sea, forest, and snow areas on the real terrain. The green and blue profiles have low and high albedo, respectively, with small contrast between sea and snow. The red profile has high contrast, with dark sea and bright snow.

virtual instrument (no changes are perceptible with or without albedo in the flux). As long as the instrument points straight to the auroral arc, the direct unpolarised light has a preponderant effect on the DoLP, which is then close to zero. When pointing to the dark sky, the DoLP is enhanced due to scattering in the atmosphere. There the DoLP is significantly affected when the albedo is taken into account. This effect is maximum in the opposite direction from the arc (Southern direction, or azimuth of $180^{\circ}$ ). For the three profiles considered in Figure 3, the choice of albedo profile seems of secondary importance. The effect of the albedo on the AoLP is of the order of $10^{\circ}$ at maximum. This occurs when pointing further away from the arc. In the following, we use the mean model (red line in Fig. 3), with albedo values of 0.2 below an altitude of $200 \mathrm{~m}$, 0.5 from $200 \mathrm{~m}$ and $500 \mathrm{~m}$, and 0.8 above $500 \mathrm{~m}$. These values match the tree line (around $400 \mathrm{~m}$ ) where the observations are performed (Skibotn, Norway).

\subsection{Inter-calibration of the sky and ground emissions}

To model the polarisation properties of one observed spectral line, POMEROL takes as input a static ground emission map that results from the integration over a wide range of wavelengths (for details, see Bosse et al., 2021). Its contribution is called $G$. Thus, for a specific spectral line of interest, we do not have access to a calibrated such map. In a wavelength outside the auroral spectrum, there may still be some light (e.g., the integrated starlight). In this case, we consider, as in Bosse et al. (2021) a uniform background source in the sky, called $K$. For the green line, the model additionally considers a calibrated all-sky image of the aurora $S_{s}(t)$ provided as a function of time. Note that for this green line, $S_{s}$ implicitly accounts for $K$. From the all-sky camera image (and the static ground albedo map), POMEROL computes a time-dependent re-emission map on the ground $S_{g}(t)$, which is inter-calibrated with the sky emissions. The contribution of the sky emissions (all-sky image) plus its reflection on the ground is then $S(t)=S_{\mathrm{s}}+S_{\mathrm{g}}$.
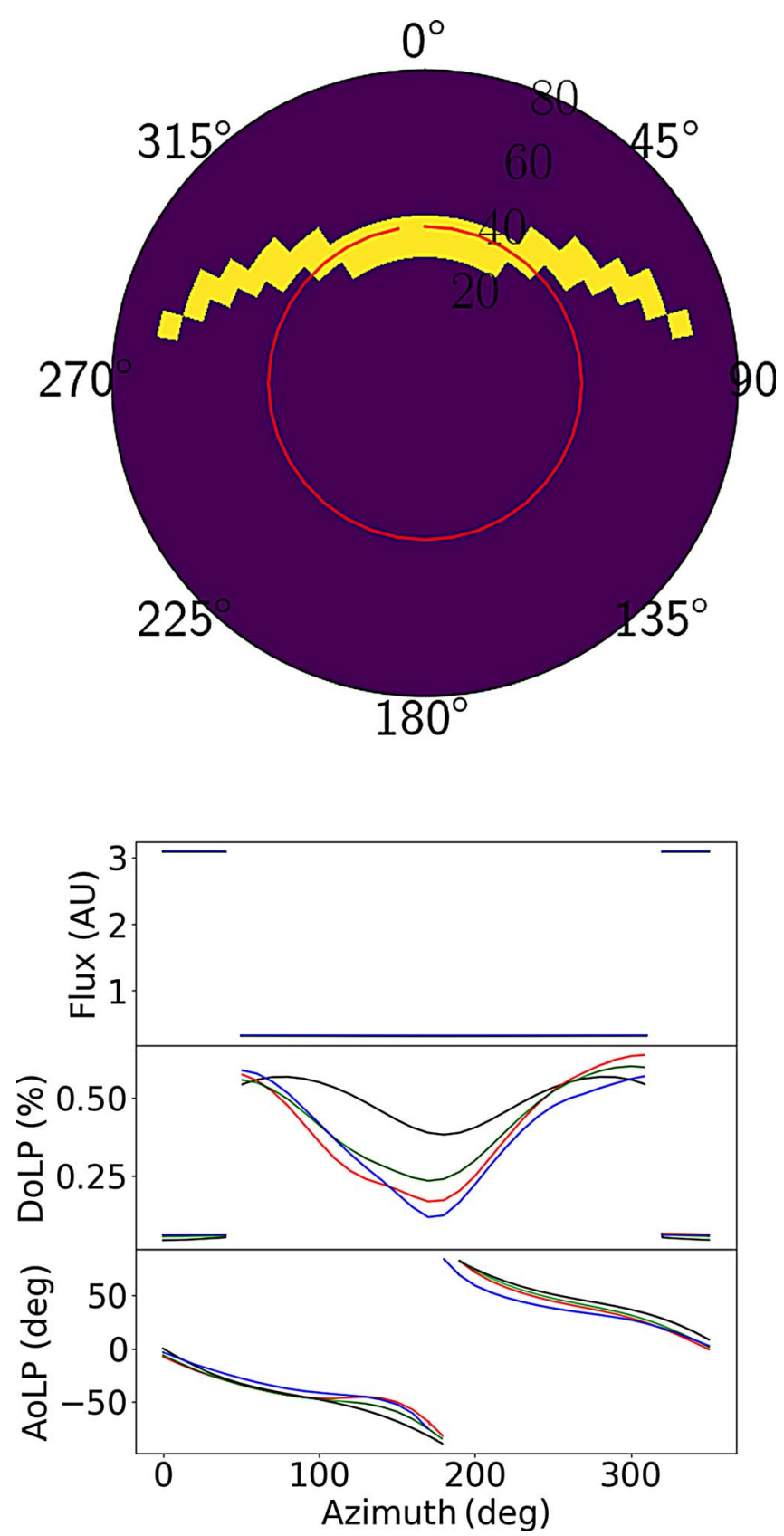

Fig. 4. Top: All-sky image for an East-West synthetic aurora spanning the sky at $45^{\circ}$ elevation Northward. The red curve shows the pointing direction of the instrument along a $45^{\circ}$ almucantar. The radiance of the aurora (in yellow) is $100 \mathrm{nW} / \mathrm{m}^{2} / \mathrm{sr}$, against $10 \mathrm{nW} /$ $\mathrm{m}^{2} / \mathrm{sr}$ for the background. Bottom: Almucantar under a synthetic aurora. Comparison using different albedo curves from Figure 3. In black: no albedo. Other colors match the profiles shown in Figure 3. Azimuths are indicated with respect to the Northern direction, positive eastward.

The model computes separately the contributions from the ground $G$, from a uniform sky $K$, and from the auroras $S(t)$. Since $S, G$, and $K$ are not inter-calibrated, one cannot simply add them together. Depending on the measured wavelength, we face several configurations: 
Table 1. List of the observations considered throughout this study.

\begin{tabular}{|c|c|c|c|c|c|c|}
\hline Line & Date & Start (UT) & Duration (mn) & Wavelength (nm) & Azimut (deg) & Elevation (deg) \\
\hline Green & $2019-3-8$ & 01:20 & 7 & 557.7 & Almucantar & 45 \\
\hline Orange & $2020-2-27$ & $22: 25$ & 6 & 620 & Almucantar & 52 \\
\hline Green & $2020-2-27$ & $22: 25$ & 6 & 557.7 & Almucantar & 52 \\
\hline Purple & $2020-2-27$ & $22: 25$ & 6 & 391.4 & Almucantar & 52 \\
\hline
\end{tabular}

- In the green line, because the all-sky camera is photographing the sky in the $557.7 \mathrm{~nm}$ band, no extra contribution from the background sky is required, and $K$ is set to zero. A linear combination $T=\alpha G+\beta S$ (with $\alpha$ and $\beta$ positive real numbers) is then performed to build the model predictions to the observed flux variations.

- In a non-auroral line (e.g., the orange at $620 \mathrm{~nm}$ ), the model predictions reduce to a linear combination of the form $T=\alpha G+\gamma K$, with $\gamma>0$ (the reflection on the ground from $K$ is ignored as we found it to be negligible compared with the light coming directly from the sky).

- For an auroral line different from the green (purple and blue lines), no all-sky pictures are available. We then derive an approximated auroral emission map from images acquired in green. In this case, the isotropic background contribution $K$ is added to $S$. The last contribution must be added as well, called $K_{\mathrm{A}}$. It is a correction of the contrast of the green input all-sky image (relative difference between the dimmest and brightest pixel of the image). This "auroral background" contribution is assumed to be part of the aurora, which can not be captured by the green all-sky camera. Its polarisation is a free parameter in the code. The total flux received by the virtual instrument is then $T=\alpha G+\beta S+\gamma K+\delta K_{\mathrm{A}}$. Note that $\delta$ can be positive or negative, respectively reducing or increasing the contrast of the green image.

This approach suffers from the fact that the ground light pollution maps are averaged (1000 pixels mapping an area of $100 \mathrm{~km}$ radius around the instrument) over a full year (2016) and integrated into wavelengths (see Bosse et al., 2021).

\section{Campaigns in auroral conditions}

A series of experiments were conducted in March 2019 and February 2020 at the Skibotn Astronomical Observatory in Norway $\left(69.34812^{\circ} \mathrm{N}, 20.36322^{\circ} \mathrm{E}\right)$. Their main characteristics are summarized in Table 1 . The 2019 records have already been reported in Bosse et al. (2020). The Skibotn observation site is located $5 \mathrm{~km}$ South-West from Skibotn. The northern conditions are difficult to predict and can vary from minutes to minutes. During these two campaigns, we could gather successful observations at different wavelengths. Unfortunately, we failed in observing the oxygen red line at $630 \mathrm{~nm}$. This is unfortunate as this wavelength was the only one observed with a former instrument (Lilensten et al., 2015). However, we could get observations at other wavelengths of interest. Some are present in the nightglow. Some are present in the urban lighting (an average spectrum of this light pollution in Skibotn is given in Bosse et al. (2020). We summarize below the main specificities for the several spectral lines considered in our study:

1. The Orange filter at $620 \mathrm{~nm} \pm 5 \mathrm{~nm}$ corresponds to $\mathrm{OH}$ emissions in the high altitude mesosphere. It exists everywhere and is assumed stationary (Bellisario et al., 2014). It is expected not to be polarised at the emission and is hardly sensitive to solar activity (Bellisario et al., 2020). It is also widely present in urban lighting.

2. The purple $(391.4 \mathrm{~nm} \pm 5 \mathrm{~nm})$ and blue $(427.8 \mathrm{~nm} \pm 5 \mathrm{~nm})$ filters correspond to bright emissions of the $1^{\text {st }} N_{2}^{+}$negative band. They constitute, therefore, ionospheric emissions originating typically between 85 and $90 \mathrm{~km}$ altitude. In the auroral zone, they are expected to be much brighter than the star emissions. Although Bosse et al. (2020) already measured an indisputable polarisation in these wavelengths, the source of this polarisation - either at the emission or by Rayleigh or Mie scattering - is not ascertained and is not documented in the scientific literature. These wavelengths are absent or very weak in the urban lights.

3. The green line is the most intense auroral line at $557.7 \mathrm{~nm}$ due to the $\mathrm{O}^{1 \mathrm{~S}} \rightarrow \mathrm{O}^{1 \mathrm{D}}$ transition. It is, therefore, a thermospheric emission, maximum around $110 \mathrm{~km}$. Following Bommier et al. (2011), it should not be theoretically polarisable. However, its polarisation has also been observed from the ground (Bosse et al., 2020), and assessing whether the polarisation is created at the emission or after the emission is a key point to better understand the behavior of the atomic oxygen. The $557.7 \mathrm{~nm}$ emission is also present in Skibotn lamps spectra, with an intensity about $75 \%$ smaller than in the $620 \mathrm{~nm}$ band. Our filter has a bandwidth of $10 \mathrm{~nm}$.

The solar activity conditions were quiet to unsettled in 2019 with $f_{10.7}$ index of 69 (https://www.spaceweather.gc.ca/ solarflux/sx-en.php) and a magnetic index $a p=12$ (http://isgi. unistra.fr/). There was some auroral activity during the full night, and the sky remained clear. The geophysical conditions were quieter in February 2020, with a decimetric index $f_{10.7}=63$, an $a p$ index varying between 2 and 3, and no auroral activity (therefore, rather stationary observation conditions). In 2019, we used a one-channel instrument. The color filters had then to be switched manually, forbidding to observe several wavelengths at the same time. In 2020, we could use a multichannel instrument. 


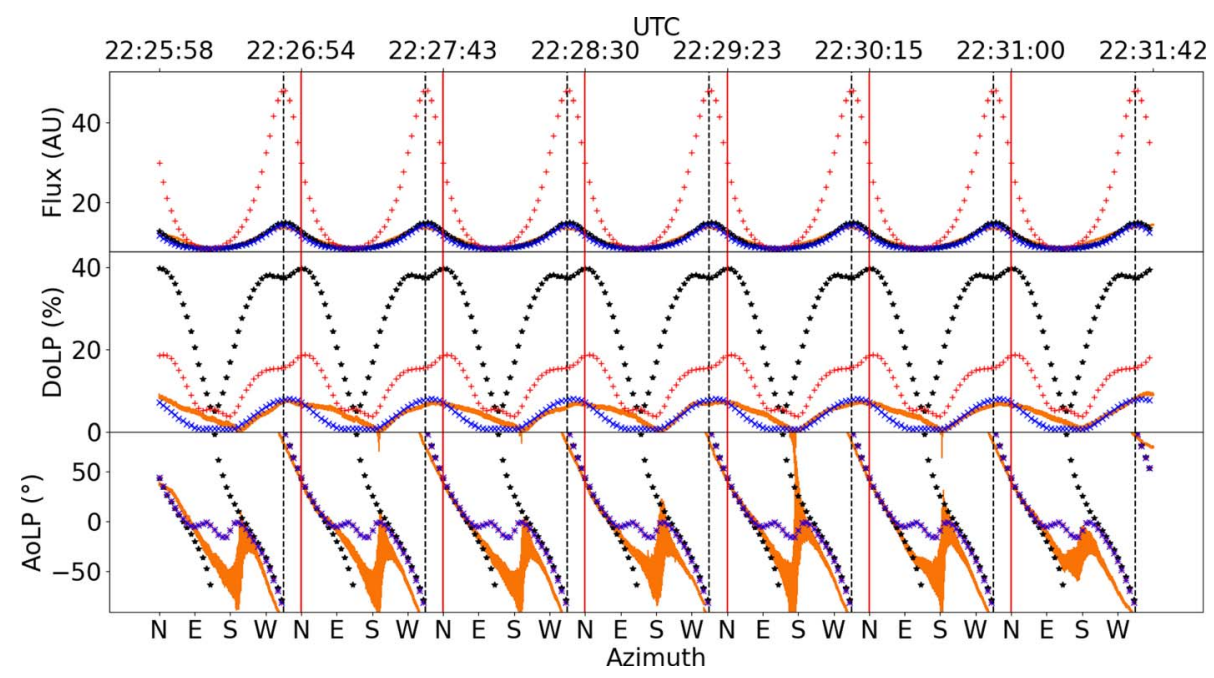

Fig. 5. In orange, the observations of the polarisation flux (top), DoLP (middle), and AoLP (bottom) at $620 \pm 5 \mathrm{~nm}$ over 7 almucantars on February 27, 2020. The thickness of the line indicates the uncertainties). A sliding average of $10 \mathrm{~s}$ is applied. Black stars ( $\star$ ): model without aerosols (no Mie scattering) nor isotropic background $(\gamma=0)$. Red plus $(+)$ : idem but model with aerosols. Blue $(\times)$ : same as red with an isotropic background set to fit the flux. The vertical dashed lines show the Skibotn direction.

\section{Results: observations versus model predictions}

Observations obtained in February 2020 gather simultaneously three wavelengths, namely Orange $(620 \mathrm{~nm})$, Green $(557.7 \mathrm{~nm})$, and Purple $(391.4 \mathrm{~nm})$. Figure A.1 shows the ground emission map used as input for the POMEROL model, together with the constant altitude lines corresponding to the albedo definition. The all-sky camera picture showing the green aurora present at the time of observations is shown in Figure A.2. The state of the auroral emissions was supposed stationary during the whole observation period (around 6 min) so that only one all-sky picture was used.

\subsection{Estimation of the aerosol model from the $620 \pm 5 \mathrm{~nm}$ observations}

We focus here on the observations with the orange filter made on February 27th, 2020, during seven successive almucantars (see Fig. 5). These almucantars were performed by hand, rotating the instrument as smoothly as possible. Each almucantar lasts around $50 \mathrm{~s}$. We recall that there is two data points per second averaged using a sliding window of $10 \mathrm{~s}$. The observed flux (top panel) maximizes above the village of Skibotn in the NW direction. The DoLP (middle panel) reaches values as high as $7.5 \pm 0.5 \%$. It peaks above Skibotn. The AoLP (bottom panel) spans twice the $\left[-90^{\circ},+90^{\circ}\right]$ range per almucantar, as expected from the Rayleigh scattering theory, with a value of $\pm 90^{\circ}$ above Skibotn and in the SSW direction.

In Figure 5, we compare the observations with the model outputs under several assumptions. When the model takes neither the background (stars and nightglow) nor the aerosols into account (but still Rayleigh scattering by the atmosphere), or $\beta=\gamma=0$, the flux reproduces the data.

The DoLP reaches values up to $40 \%$ around the northern direction, with a double peak signature, indicating a predominant point source located in the direction of Skibotn (see Bosse et al., 2021). The AoLP rotates regularly with the azimuth and fits the data very well except between the East and South-West directions.

Adding aerosol scattering into the model changes drastically the model predictions. The amplitudes of the flux variations increases by a factor of about 3: from 8 to 14 (resp. 8 to 47, in arbitrary units) without (resp. with) aerosols. The DoLP variations along one almucantar are divided by 2, peaking at values around 20\% around the Skibotn direction, still significantly larger than the observations. The AoLP is modified only in the direction of the unidentified contribution. It does not fit the data in this direction better than without aerosols.

Finally, combining the aerosol scattering and the isotropic background $(\gamma>0)$, we obtain the last model. The background flux is computed to fit the flux variations with a value of $43 \mathrm{AU}$ (effectively doubling the average model flux). The DoLP is again reduced by a factor $\approx 2$, with a peak now at $8 \%$ in the direction of Skibotn, very similar to the observations. The modeled DoLP fits the data except around the eastward direction, where we suspect an unidentified contribution. Because the background is unpolarised, the AoLP is the same as that predicted by the model with aerosols but without background.

In Figure 6, we show a zoom on the best fit of the model. We tested a series of aerosol parameters. They are characterized by their complex refractive index, size distributions, and vertical profiles. The full discussion provided in Bosse et al. (2021) is not repeated here. The three models used in this previous study of mid-latitude observations were unsuccessful to reproduce the observations. We adjusted the "1-low" aerosol model by Bosse et al. (2021) with a new complex refractive index of $1.6+i 0.0035$. A real part of 1.6 is a high refractive index but still in the range of typical values (from 1.4 to 1.7 , see Ebert et al., 2004; Dubovik et al., 2000; Lenoble et al., 2013). The other parameters define the log-normal size distribution of the aerosols with a mode radius $R=150 \mathrm{~nm}$ and a dispersion $\mathrm{ln}$ $(\sigma)=0.29$, as well as the vertical concentration profile with a 


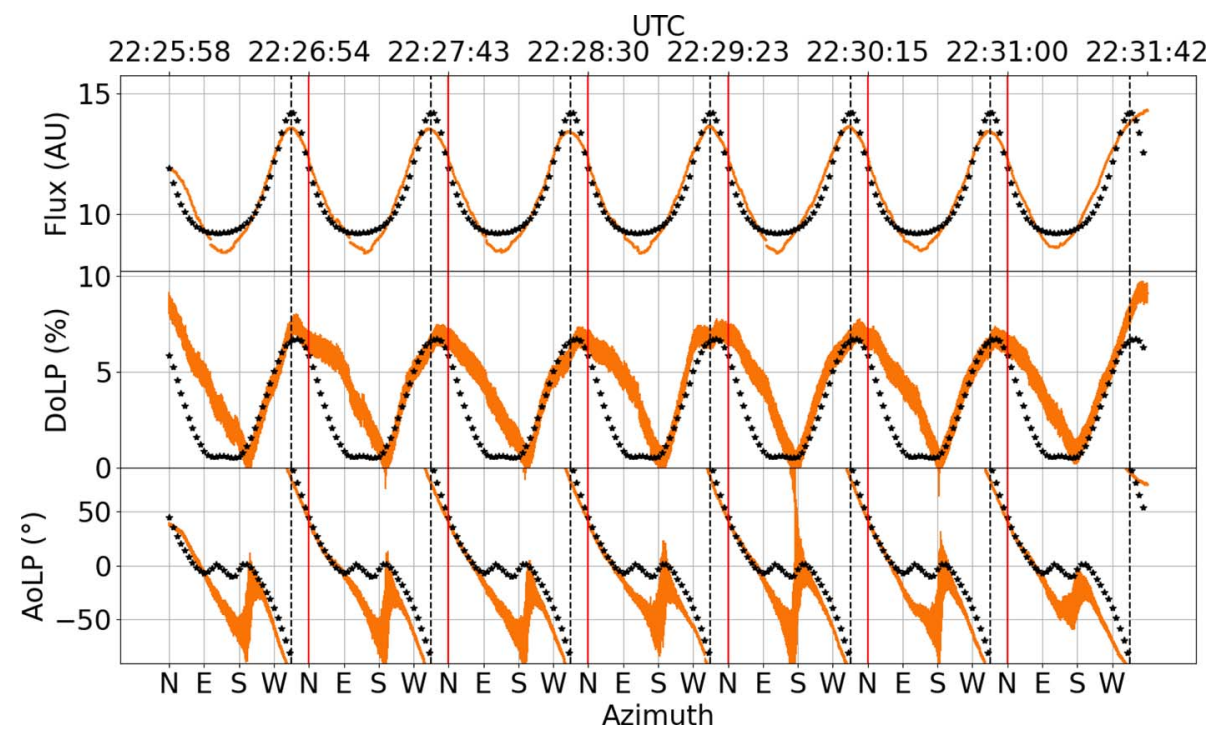

Fig. 6. Same as Figure 5 for the aerosols profile offering the best fit to the data (see text for details).

scale height $H=440 \mathrm{~m}$, a ground concentration $n_{0}=4000 \mathrm{~cm}^{-3}$ and a background concentration $n_{\mathrm{B}}=10 \mathrm{~cm}^{-3}$. We cannot state that our choice is the unique combination that would fit the data. For example, lowering the refractive index can be compensated with a higher concentration and vice-versa. However, the modeled DoLP and AoLP are highly dependent on the aerosol size distribution, which is thus better constrained.

At this stage, the comparison at $620 \mathrm{~nm}$ between modeling and observations allows drawing some conclusions:

- A reasonable aerosol model from the fit between the modeled and measured flux and DoLP. This solution is probably not unique, but this paves the way for an original and promising approach to characterize the aerosols (size distribution, concentration, optical index).

- The relative intensity of the background flux in the orange line.

- The existence of an additional light source that does not exist in the light pollution maps in the SE direction. This unidentified source (which may be ephemeral) is evidenced through its effects on the flux.

\subsection{Observations in the green line require a polarisation at the source}

We now have a good estimate of the aerosol model, which we adopt for all other observations. At $557.7 \mathrm{~nm}$, we can also make use of all-sky camera observations to determine the total sky intensity $S$. In the polariser, this source is merged with that of the nearby village $G$. The combination of those two contributions on the instrument is shown in Figure 7 and compared to observations over 7 almucantars. We adjust the linear combination (values of $\alpha$ and $\beta$ ) in order to best fit the flux observations in the same approach as for the orange above. This gives $30 \%$ for $G$ the ground contribution and $70 \%$ for $S$ the sky one. However, this proportion is badly constrained, and the ground contribution can be decreased to values as low as $20 \%$ without impacting significantly the fit of the flux. In all cases, the resulting computations of the DoLP and AoLP show a strong discrepancy with the measurements. First, the AoLP is always controlled by that of the village, with a strong phase shift over one almucantar. Second, the modeled DoLP maximum is largely overestimated in comparison with the measured DoLP. Furthermore, the measured DoLP peaks in the SE direction, while the DoLP of the linear combination peaks in the Western direction. In the green line, incorporating the effect of multiple scattering in the atmosphere should tend to only slightly decrease the DoLP. However, we do not see how it could shift the peak of DoLP, nor rotate the AoLP enough to correct for the observed discrepancies. Furthermore, as was the case for both the green and orange lines observed at mid-latitude (Bosse et al., 2020), auroral observations in the orange line can be interpreted without a proper account of multiple scattering. We thus see no reason for it to be necessary for the green line, and the discrepancies must come from another source. Thus, two conclusions can be drawn from this study:

- In the absence of a polarised source, it is the contribution from ground light pollution that controls the DoLP and AoLP modeled along one almucantar. Contrary to what is observed at $620 \mathrm{~nm}$, we cannot fit the observed polarisation measurements in the green line this way.

- In order to better model the observed DoLP and AoLP, we need to add another contribution. The only option that we foresee is to add a direct polarisation produced in the upper atmosphere.

We then perform a second linear combination between the different sources, adding a polarised contribution from the thermospheric green line (Fig. 7, blue curve). Since the green line is emitted in the conductivity layer, we assume that this polarisation is due to electron collisions with the atomic oxygen, the impacting electrons are a combination of horizontal currents (Hall and Pedersen), with a possible vertical motion along the magnetic line. We consider a minimal model with only three additional parameters: the direction of the electron flux (azimuth 


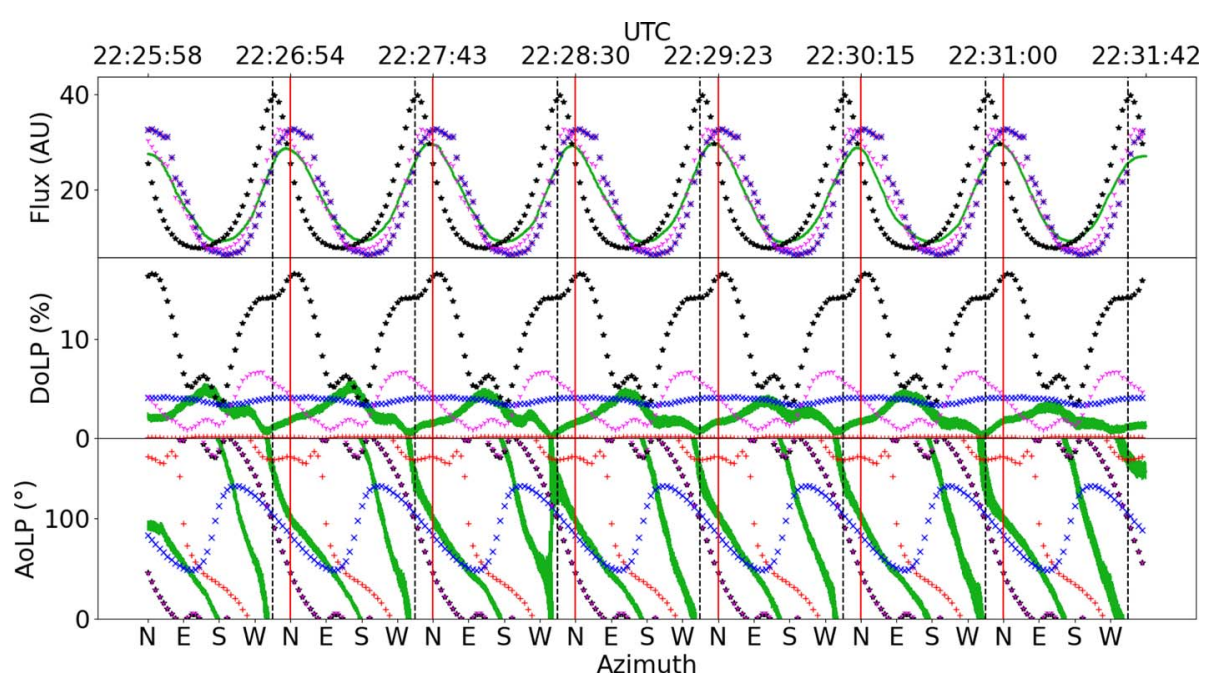

Fig. 7. In green, the observations of the polarisation flux (top), DoLP (middle), and AoLP (bottom) at $557.7 \pm 5 \mathrm{~nm}$ over 7 almucantars on February 27, 2020. The thickness indicates the uncertainties). A sliding average of $10 \mathrm{~s}$ is applied. Black stars ( $\star$ ): Contribution of the ground emissions $G$. Red plus (+): Contribution of the sky emission and its reflection on the snow $S$. Pink (Y): Linear combination of the ground and sky contributions that best fits the flux variations. Blue crosses $(\times)$ : Direct light only (i.e., not scattered in the atmosphere, no other sources that could be scattered by the atmosphere are taken into account) with a polarisation at the emission (here $d_{\max }=5 \%$ and the electrons motion direction is $50^{\circ}$ azimuth and $-20^{\circ}$ elevation). The dashed vertical black line indicates the direction of Skibotn. The picture of the sky at $557.7 \mathrm{~nm}$ at the time of the observation is shown in Appendix A.

and elevation) and a maximum DoLP at the source. All three are supposed to be constant over the whole sky during the whole observation. The numerical method for the search of the model parameters is detailed in Appendix B. We show in Figure 8 the final model using one of the best set of parameters that we could find with a non-systematic search. It is likely that this solution is not unique. A systematic numerical search for the best fit is out of the scope of this paper: what we aim at showing is that the observations cannot be modeled without a polarisation source in the upper atmosphere. For the solution illustrated here, the village contribution is $20 \%$, against $80 \%$ for the sky. Within this latter, a partial polarisation with a $d_{\max }=5 \%$ is found. The motion of the electrons at the source of the emission is set with an azimuth of $50^{\circ}$ and $-20^{\circ}$ elevation (North-East, downwards). Remains the issue of the phase function describing in which plane the angle of polarisation occurs. In the absence of any information on this parameter, we use the same as for the Rayleigh scattering. The DoLP of a light beam scattered at an angle $\theta$ is (McFarlane, 1974)

$$
\operatorname{DoLP}(\theta)=d_{\max } \frac{\sin ^{2} \theta}{1+\cos ^{2} \theta} .
$$

The associated AoLP is determined by the direction perpendicular to the plane defined by the incoming electrons (potentially a current) and the emitted light beams.

In Figure 8, the modeled intensity (top panel) fits reasonably well the measurements. The modeled DoLP (middle panel) is in the same range as the measured one. It furthermore exhibits, as the observations, two maxima over each almucantar. One (in the southern direction) is reasonably well reproduced, while the second one (in the NW direction) is shifted and overestimated when compared to the observed DoLP. The modeled AoLP reproduces relatively well the measured one excepted in the SW quadrant where we fail in reproducing the magnitude of the second maximum in the DoLP.

From this comparison, several conclusions can be drawn:

- The confrontation of the POMEROL model and the observations requires a green $(557.7 \mathrm{~nm})$ thermospheric auroral emission that is polarised at the source.

- In the circumstances of the 2020 campaign, this polarisation peaks at about $5 \%$.

- The source of the excitation prevailing to this polarised emission is mainly horizontal, suggesting ionospheric currents. A vertical component exists as well, possibly explained by a component of the electron trajectory along the magnetic field lines.

\subsection{Observations in the purple line require a polarisation at the source}

We now consider observation in the purple line (391.4 $\mathrm{nm} \pm 5 \mathrm{~nm}$ ), recorded simultaneously as the orange and green lines above. We follow the same approach as the green line detailed in the previous section. Again, it turns out to be impossible to fit the model and the measurements without considering a polarisation at the source. To estimate it, we use the same current parameters as found for the green line since the purple emission occurs at the bottom of the high conductivity layer. In this case, the best fitting maximum DoLP value for the source is $d_{\max }=11 \%$, larger than that of the green line, for the same electron direction. The results of the best fit are shown in Figure 9. The contribution of the village on the intensity is now about $10 \%$. The predicted flux nicely reproduces the 


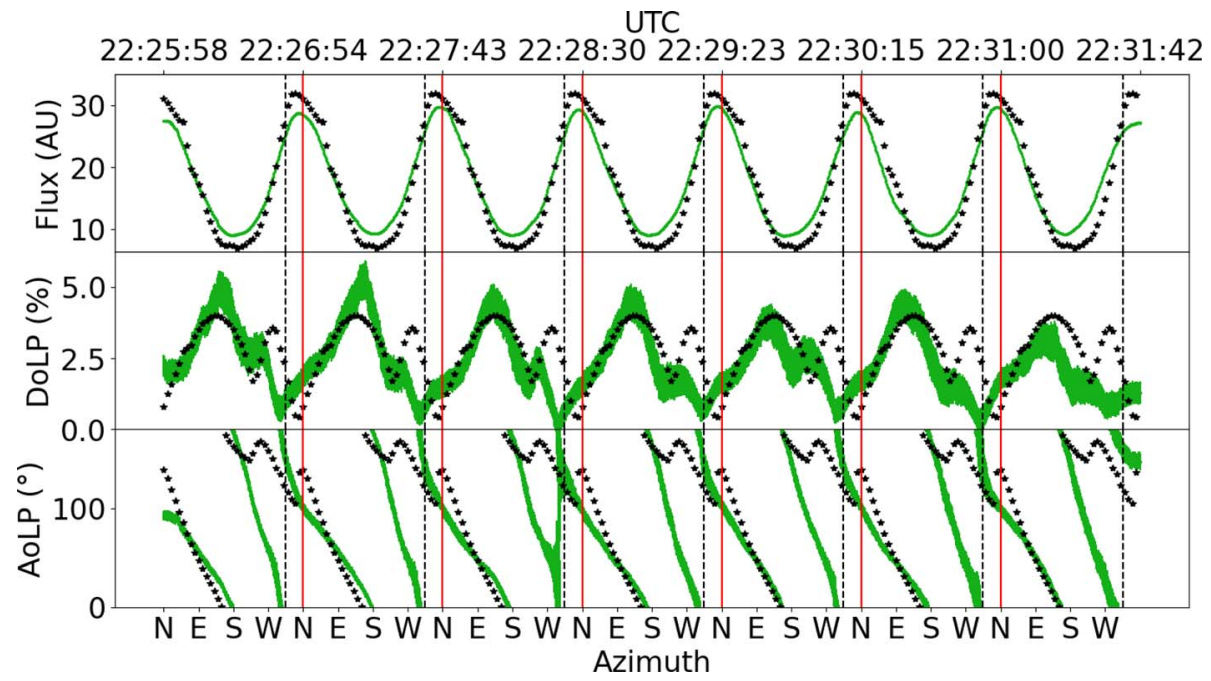

Fig. 8. 7 almucantar observations at $557.7 \pm 5 \mathrm{~nm}$ (February 27, 2020): observations (in green) and predictions for the model that best fits the data (Black stars $\star$ ). The model is a linear combination of ground and sky (including albedo) for a $5 \%$ polarised sky emission in a direction defined by an azimuth of $50^{\circ}$ and $-20^{\circ}$ elevation (see text for details). The dashed vertical black line indicates the direction of Skibotn. The picture of the sky at $557.7 \mathrm{~nm}$ is shown in Appendix A.

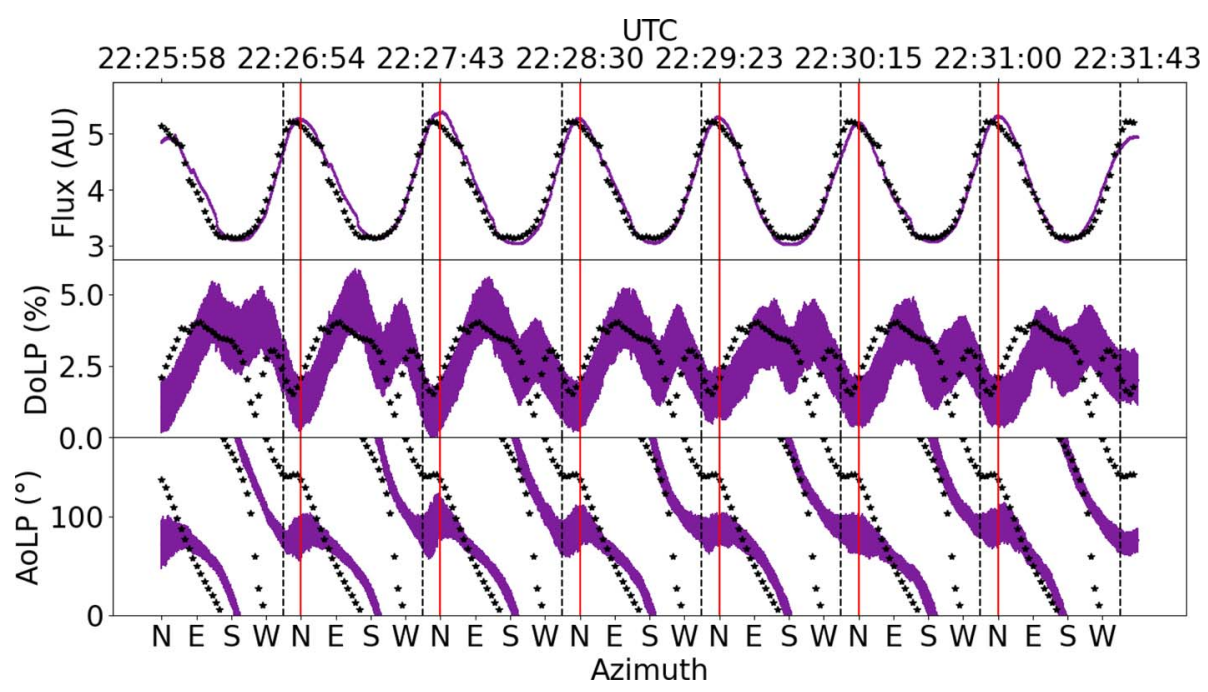

Fig. 9. 7 almucantar observations at $391.4 \pm 5 \mathrm{~nm}$ (February 27, 2020): observations (in purple) and predictions for the model that best fits the data (Black stars $\star$ ). The model is a linear combination of ground and sky (including albedo) for a $11 \%$ polarised sky emission in a direction defined by an azimuth of $50^{\circ}$ and $-20^{\circ}$ elevation (see text for details). The dashed vertical black line indicates the direction of Skibotn. The picture of the sky at $557.7 \mathrm{~nm}$ is shown in Appendix A. The emissions at $391.4 \mathrm{~nm}$ (purple) are considered to be similar.

observed one despite a weak contribution from the city lights pollution. This is coherent with the fact that the purple intensity is smaller in the village lamps than the green and orange ones. Since we used the green line all-sky camera as sky input, this shows that in this particular event, the dynamics were likely close at $110 \mathrm{~km}$ (green emission) and at $85 \mathrm{~km}$ (purple emission).

The modeled and observed DoLP not only range within the same magnitudes. The model also replicates the two peaks observed by the polarimeter over one almucantar. The minimum to the North is well reproduced, while that to the SW is shifted. Given the simplicity of the source model that we considered (a single orientation for the polarisation at the source), the obtained fit is remarkable.
The modeled AoLP (bottom panel) fails in reproducing the data in the Western quadrant. This direction corresponds to the minimum DoLP, i.e., the ALP data are the noisiest (Bosse et al., 2020). In other directions, the model predictions and observations are overall coherent. Note that the fit to the data might still be improved with a more sophisticated model of the polarisation at the emission. The conclusions are:

- The confrontation of the POMEROL model and the observations requires a purple $(391.4 \mathrm{~nm})$ ionospheric auroral emission that is polarised at the source.

- This polarisation maximizes at about $11 \%$, i.e., it is apparently larger than that of the green line. 


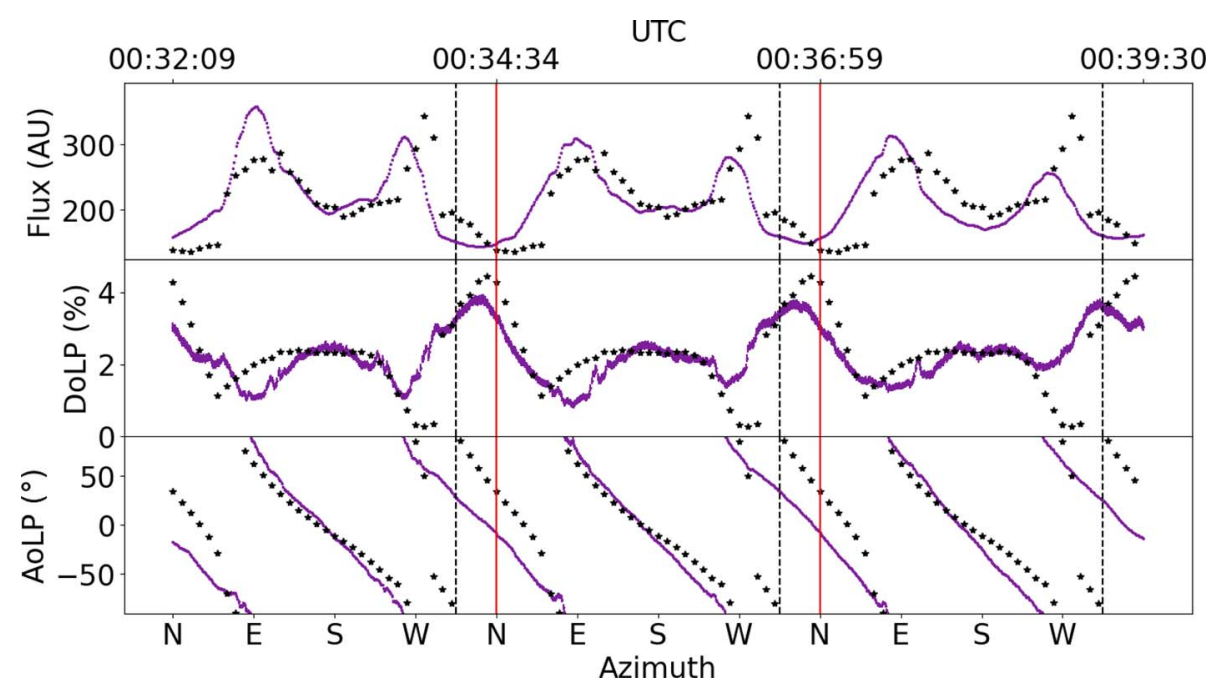

Fig. 10. 3 almucantar observations at $391.4 \pm 5 \mathrm{~nm}$ (March 8, 2019): observations (in purple) and predictions for the model that best fits the data (Black stars $\star$ ). The model is a linear combination of ground and sky (including albedo) for a $3 \%$ polarised sky emission in a direction defined by an azimuth of $35^{\circ}$ and $-3^{\circ}$ elevation (see text for details). The dashed vertical black line indicates the direction of Skibotn. The picture of the sky at $557.7 \mathrm{~nm}$ is shown in Appendix A. The emissions at $391.4 \mathrm{~nm}$ (purple) are considered to be similar.

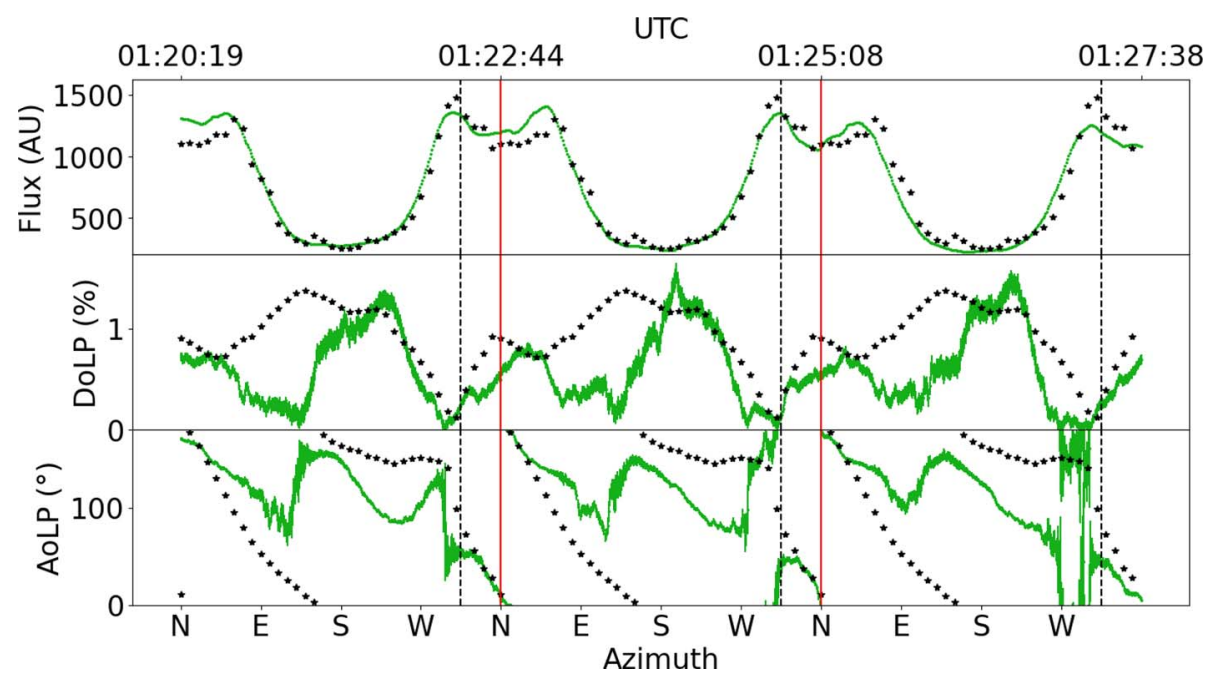

Fig. 11. 3 almucantar observations at $557.7 \pm 5 \mathrm{~nm}$ (March 8, 2019): observations (in green) and predictions for the model that best fits the data (Black stars $\star$ ). The model is a linear combination of ground and sky (including albedo) for a $1.3 \%$ polarised sky emission in a direction defined by an azimuth of $35^{\circ}$ and $-3^{\circ}$ elevation (see text for details). The dashed vertical black line indicates the direction of Skibotn. The picture of the sky at $557.7 \mathrm{~nm}$ is shown in Appendix A.

\subsection{Model versus observations under intense auroral activity}

We now turn to the observations collected in 2019 under more active auroral conditions. This series of measurements have been described in Bosse et al. (2020). We were not able to measure the emissions simultaneously. We could perform sequentially observations in the purple, green, and blue lines (respectively Figures 10, 11, and 12). We only show below the best fits between the observations and the POMEROL model outputs over three almucantars. No observations outside the auroral emissions (e.g., orange at $620 \mathrm{~nm}$ ) are available for the 2019 campaign. Without further information, we thus use the aerosol model derived from the 2020 orange observation. The main interest in showing these measurements lies in the auroral conditions, which were very active, with a bright and dynamic aurora (while the sky was very dark during the February 2020 campaign documented above). The all-sky camera used as input for modeling the green and purple almucantars is shown in Figure A.3. The aurora extends from East to West in a series of curtains. This results in two maxima in the measured flux per almucantar.

To obtain the fit shown in Figure 10 for the purple line, the village contribution is $10 \%$, similar to that of 2020 , in spite of the fact that the purple line intensity looks much higher in 2019 than in 2020. However, Figure 10 shows the flux in 


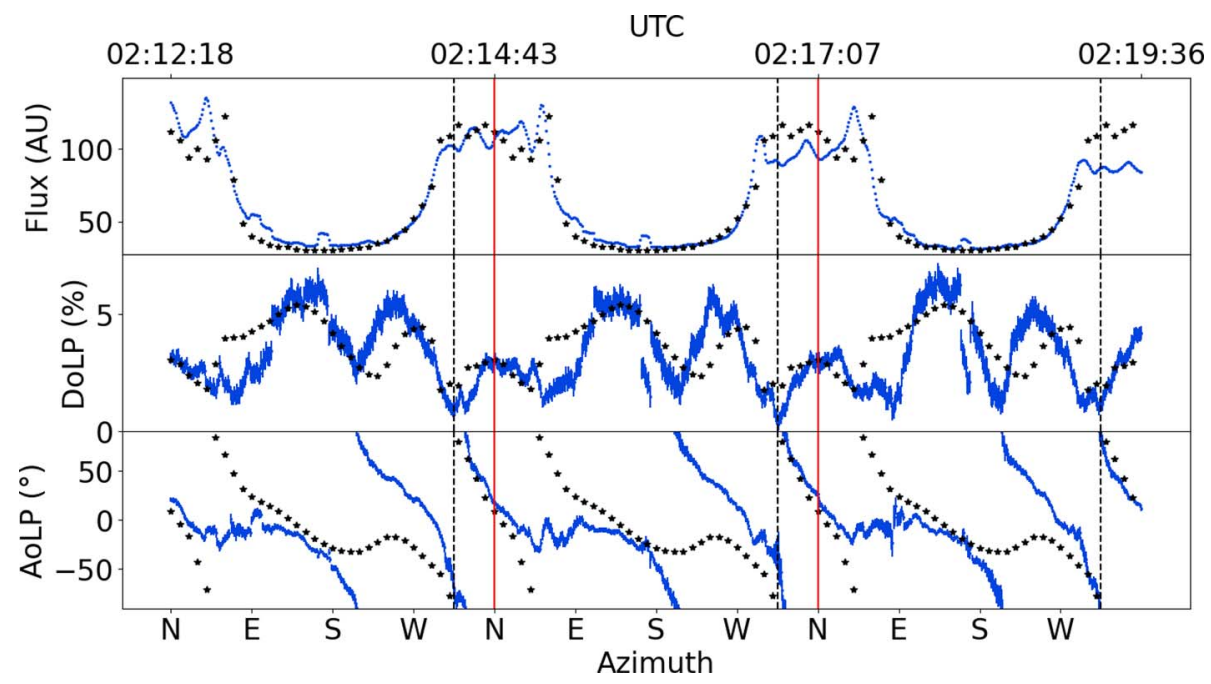

Fig. 12. 3 almucantar observations at $427.8 \pm 5 \mathrm{~nm}$ (March 8, 2019): observations (in blue) and predictions for the model that best fits the data (Black stars $\star$ ). The model is a linear combination of ground and sky (including albedo) for a $6 \%$ polarised sky emission in a direction defined by an azimuth of $50^{\circ}$ and $10^{\circ}$ elevation (see text for details). The dashed vertical black line indicates the direction of Skibotn. The picture of the sky at $557.7 \mathrm{~nm}$ is shown in Appendix A.

arbitrary units since the photomultipliers were different from one year to another, with no inter-calibration. Moreover, the absence of all-sky camera at $391.4 \mathrm{~nm}$ prevents intercomparing the intensities of these different aurorae. It is then hazardous to go further in the interpretation of this number. The direct polarisation of the purple line is smaller than that obtained in the quieter case in 2020, maximizing at only $d_{\max }=3 \%$. The comparison of modeling and observation of the polarisation parameters in the green line is shown in Figure 11. The village contribution is reduced to $7.1 \%$, which is coherent with the fact that the green thermospheric line is very bright, and the maximum source polarisation is now $d_{\max }=1.3 \%$. For the blue line (Fig.12) the model uses a maximum source polarisation of $d_{\max }=5.9 \%$ and a ground contribution of $26 \%$. For the purple, blue and green lines, the best fitting electron incoming direction are close: respectively $249^{\circ}, 232^{\circ}$, and $244^{\circ}$ in azimuth and $2.3^{\circ}, 0^{\circ}$, and $5^{\circ}$ in elevation, i.e., almost horizontal, South-West (or North-East, as the direction is symmetrical). In the purple and blue lines, the flux and the DoLP are remarkably well reproduced by the model, in spite of the dynamics of the aurora. The AoLP is well reproduced for the purple line but not for the blue. The comparison is less convincing in the green line, with some misfit for the DoLP in the Eastern direction and some shift in the AoLP in the SW quadrant.

The above analysis shows that the polarisation rate at the emission required to fit the observations is higher in quiet geomagnetic conditions than in active conditions. Two competing mechanisms may explain it: (i) the polarisation at the source may be due mainly to low energy electrons impacting the atmospheric targets. In the 2020 case, during a quiet night, these electrons constituted a major part of the incoming precipitation. They were still present in 2019 during an active auroral event but in a proportionally smaller quantity. (ii) The polarisation of the emissions is the same, whatever the impacting electron energy, but there are more depolarisation due (e.g.) to collisions during active conditions.

\section{Summary of the main results}

Using an improved version of the POMEROL radiative transfer model by Bosse et al. (2021), we have presented an analysis of night sky polarisation observations at auroral latitudes during two dedicated campaigns in March 2019 and February 2020. This comparison allows separating the effects of the light pollution due to the scattering in the lower atmosphere from that of the natural skylight. We showed that:

1. This technique is a proxy of aerosol properties (refractive index, size, and number density) in the lower atmosphere by using a mesospheric line (here, $\mathrm{OH}$ at $620 \mathrm{~nm}$ ) as background skylight.

2. The confrontation of the model predictions with the observations requires that the green (thermospheric), blue and purple (ionospheric) auroral emissions are polarised.

3 . The polarisation angle seems associated with the interaction between the atmospheric targets and the incoming electrons. The incoming electron motion has both vertical (along the magnetic field lines) and horizontal (due to the ionospheric currents) components. If confirmed, the angle of polarisation can become a unique way to measure the ionospheric currents in real-time from the ground, a parameter of great interest in many space weather applications.

4. The polarisation rate is larger in quiet auroral conditions, showing that either low energy electrons are more efficient to create the polarisation or that depolarisation is taking place in active conditions. If confirmed, the DoLP may become a way to estimate the energy of the incoming electrons (precipitated or in the ionospheric currents), another important space weather parameter.

5. The purple line polarisation rate (DoLP) seems larger than that of the green line. This conclusion, however, depends significantly on the best fit criterium (detailed in Appendix B) and ought to be confirmed with further experiments and a systematic search for the best fit. 
Our study, which shows the necessity to consider auroral lights as polarised, should be considered a first attempt. More experiments are necessary to confirm the above conclusions. In particular, the search for optimal solutions requires an improved strategy (the model predictions are nonlinearly related to the input parameters). Further experimental and numerical efforts should allow making the determination of the aerosol parameters more automatic (conclusion 1). As it is already done during the day-time (Hasekamp, 2010; Snik et al., 2014), nighttime polarisation measurements along with the POMEROL model could be used to determine atmospheric aerosol composition. This would require a thorough validation and calibration process using independent aerosol measurements. They should also help to better determine what electron parameters (energy, direction) can be drawn from the polarisation measurements (conclusions 2, 4, and 5). In particular, observing the same emission volume from different locations with three polarimeters will be of great help.

Of course, our model remains imperfect, in particular as it does not account properly for multiple scattering, a mechanism that should be implemented in the future. Indeed, its effect on the DoLP may help reduce the discrepancies between the model predictions and the observations after the addition of the direct polarisation. However, we argue that to explain our observations, multiple scattering in the atmosphere is unlikely to replace a polarisation originating in the upper atmosphere (conclusion 2). Indeed, the needed correction must increase and/or decrease the DoLP and turn the AoLP, depending on the observed azimuth. Thus, this complex behavior can hardly be due to the multiple scattering addition alone.

The confrontation of the POMEROL model with our observations leads us to propose that the origin of the auroral emission polarisation is intimately related to the preferential motion direction of the electrons in the upper atmosphere (conclusions 2 and 3). However, the exact physical mechanism producing such a polarisation remains uncertain. For the sake of the demonstration, we considered here a very simple model for this polarised emission, which should be further explored in the future. For this purpose, a better comprehension of the upper atmospheric processes producing such polarisation is needed. We foresee two possibilities: the polarisation is produced directly at the emission, during the deexcitation process, or it is acquired while the light ray travels across ionospheric currents. Both hypotheses can co-exist, and the question is then to know the contribution of each effect on the polarisation and how this can bring new information on the upper atmosphere.

Conclusion 3 is supported by the fact that in previous measurements (Bosse et al., 2020) the AoLP in different wavelengths were found to be sometimes parallel to the magnetic field line. In order to confirm and eventually definitely prove this point 3 , we need simultaneous red, green, blue, and purple line measurements. Experiments with measurements of the AoLP in the red line $(630 \mathrm{~nm})$ are also requested, because this emission occurs above $110 \mathrm{~km}$, where there is hardly any horizontal current. Finally, we also need to introduce the currents measured by other instruments (magnetometer, Incoherent Scatter radar) since the model used here (with a uniform direction) is probably too simple to represent the auroral ionosphere dynamics (Pulkkinen et al., 2003). These will be the topics for a continuation of this polarisation study.
Acknowledgements. This work was partially funded by the IPEV project on the high latitude polarisation of the auroral emissions POLARLIS (project number 1026). It was also funded by the Prematuration CNRS program and by the Maturation program CM180023, project PTCU Number $180018 \mathrm{M}$ from the SATT company Linksium. This work was supported by the Programme National PNST of CNRS/ INSU co-funded by CNES and CEA. The polarimeter is patented by CNRS and valorized by Linksium (international patent number 1873378). We thank the EISCAT board, who selected our proposal for the invited science program PP. EISCAT is an international association supported by research organizations in China (CRIRP), Finland (SA), Japan (NIPR and ISEE), Norway (NFR), Sweden (VR), and the United Kingdom (UKRI). We also thank the University of Oslo for providing the all-sky imager data. The editor thanks two anonymous reviewers for their assistance in evaluating this paper.

\section{References}

Akmaev RA. 2011. Whole atmosphere modeling: connecting terrestrial and space weather. Rev Geophys 49(4): https://doi.org/ 10.1029/2011RG000364.

Appleton EV. 1924. Geophysical Influences on the Transmission of Wireless Waves. Proc Phys Soc 37(1): 16D-22D. https://doi.org/ 10.1088/1478-7814/37/1/312.

Bellisario C, Keckhut P, Blanot L, Hauchecorne A, Simoneau P. 2014. $\mathrm{O}_{2}$ and $\mathrm{OH}$ night Airglow emissions derived from GOMOSEnvisatInstrument. J Atmos Ocean Technol 31(6): 1301-1311. https://doi.org/10.1175/JTECH-D-13-00135.1.

Bellisario C, Simoneau P, Keckhut P, Hauchecorne A. 2020. Comparisons of spectrally resolved nightglow emission locally simulated with space and ground level observations. J Space Weather Space Clim 10: 21. https://doi.org/10.1051/swsc/ 2020017.

Bommier V, Sahal-Bréchot S, Dubau J, Cornille M. 2011. The theoretical impact polarization of the O I $6300 \AA$ red line of Earth aurorae. Ann Geophys 29(1): 71-79. https://doi.org/10.5194/ angeo-29-71-2011.

Bosse L, Lilensten J, Gillet N, Brogniez C, Pujol O, Rochat S, Delboulbé A, Curaba S, Johnsen MG. 2021. Night light polarization: modeling and observations of light pollution in the presence of aerosols and background skylight or airglow. Submitted to: J Geophys Res. https://hal.archives-ouvertes.fr/hal-03550521.

Bosse L, Lilensten J, Gillet N, Rochat S, Delboulbé A, et al. 2020. On the nightglow polarisation for space weather exploration. J Space Weather Space Clim. https://doi.org/10.1051/swsc/2020036.

Chamberlain J. 1959. On the polarisation of the oxygen red line in aurorae. Planet Space Sci 2(1): 73-75. https://doi.org/10.1016/ 0032-0633(59)90063-7.

Chisham G, Lester M, Milan SE, Freeman MP, Bristow WA, et al. 2007. A Decade of the Super Dual Auroral Radar Network (SuperDARN): Scientific achievements, new techniques and future directions. Surv Geophys 28(1): 33-109. https://doi.org/10.1007/ s10712-007-9017-8.

Dubovik O, Smirnov A, Holben BN, King MD, Kaufman YJ, Eck TF, Slutsker I. 2000. Accuracy assessments of aerosol optical properties retrieved from Aerosol Robotic Network (AERONET) Sun and Sky radiance measurements. J Geophys Res Atmos 105(D8): 9791-9806. https://doi.org/10.1029/2000JD900040. 
Duncan R. 1959. Polarisation of the red oxygen auroral line. Planet Space Sci 1(2): 112-IN3. https://doi.org/10.1016/0032-0633(59) 90006-6.

Ebert M, Weinbruch S, Hoffmann P, Ortner HM. 2004. The chemical composition and complex refractive index of rural and urban influenced aerosols determined by individual particle analysis. Atmos Environ 38(38): 6531-6545. https://doi.org/10.1016/j. atmosenv.2004.08.048.

Finlay CC, Lesur V, Thébault E, Vervelidou F, Morschhauser A, Shore R. 2017. Challenges handling magnetospheric and ionospheric signals in internal geomagnetic field modelling. Space Sci Rev 206(1): 157-189. https://doi.org/10.1007/s11214-016-0285-9.

Harang L. 1933. Eine Untersuchung der Polarisation des Nordlichtes. Zs. Geophysik 9: 162-164.

Harang O. 1960. An investigation of the state of polarisation of the red and green 3-lines in polar aurorae. Tech. Rep. Oslo University, Norway.

Hasekamp OP. 2010. Capability of multi-viewing-angle photopolarimetric measurements for the simultaneous retrieval of aerosol and cloud properties. Atmos Meas Tech 3(4): 839-851. https://doi.org/10.5194/amt-3-839-2010.

Henderson-Sellers A, Wilson MF. 1983. Surface albedo data for climatic modeling. Rev Geophys 21(8): 1743. https://doi.org/ 10.1029/RG021i008p01743.

Lehtinen M, Markkanen J, Väänänen A, Huuskonen A, Damtie B, Nygrén T, Rahkola J. 2002. A new incoherent scatter technique in the EISCAT Svalbard radar. Radio Sci 37(4): 1-15. https://doi.org/ 10.1029/2001RS002518.

Lenoble J, Remer L, Tanre D, (Eds.). 2013. Aerosol remote sensing. Springer-Verlag, Berlin Heidelberg ISBN 978-3-642-17724-8. https://doi.org/10.1007/978-3-642-17725-5.

Lilensten J, Bommier V, Barthélemy M, Lamy H, Bernard D, Moen JI, Johnsen MG, Løvhaug UP, Pitout F. 2015. The auroral red line polarisation: modelling and measurements. J Space Weather Space Clim 5: A26. https://doi.org/10.1051/swsc/2015027.
Lilensten J, Simon C, Barthélémy M, Moen J, Thissen R, Lorentzen DA. 2006. Considering the polarization of the oxygen thermospheric red line for space weather studies. Space Weather 4(11): https://doi.org/10.1029/2006SW000228.

Lv Y, Sun Z. 2014. The reflectance and negative polarization of light scattered from snow surfaces with different grain size in backward direction. J Quant Spectrosc Radiat Transf 133: 472-481. https://doi.org/10.1016/j.jqsrt.2013.09.010.

McFarlane SC. 1974. A Bethe theory for the polarization of impact radiation. J Phys B At Mol Phys 7(13): 1756-1771. https://doi.org/ 10.1088/0022-3700/7/13/021.

Miller SD, Straka W, Mills SP, Elvidge CD, Lee TF, Solbrig J, Walther A, Heidinger AK, Weiss SC. 2013. Illuminating the capabilities of the Suomi National Polar-Orbiting Partnership (NPP) Visible Infrared Imaging Radiometer Suite (VIIRS) day/night band. Remote Sens 5(12): 6717-6766. https://doi.org/10.3390/rs5126717.

Mills S, Weiss S, Liang C. 2013. VIIRS Day/Night Band (DNB) stray light characterization and correction. In: SPIE Optical Engineering + Applications, 88661P. Butler JJ, Xiong XJ, Gu $\mathrm{X}$, (Eds.) San Diego, California, United States. https://doi.org/ 10.1117/12.2023107.

Nash SG. 1984. Newton-type minimization via the Lanczos method. SIAM J Numer Anal 21(4): 770-788. https://doi.org/10.1137/ 0721052.

Pulkkinen A, Amm O, Viljanen A. 2003. Ionospheric equivalent current distributions determined with the method of spherical elementary current systems. J Geophys Res Space Phys 108(A2): https://doi.org/10.1029/2001JA005085.

Snik F, Rietjens JHH, Apituley A, Volten H, Mijling B, et al. 2014. Mapping atmospheric aerosols with a citizen science network of smartphone spectropolarimeters. Geophys Res Lett 41(20): 73517358. https://doi.org/10.1002/2014GL061462. 


\section{Appendix A: Environment maps}

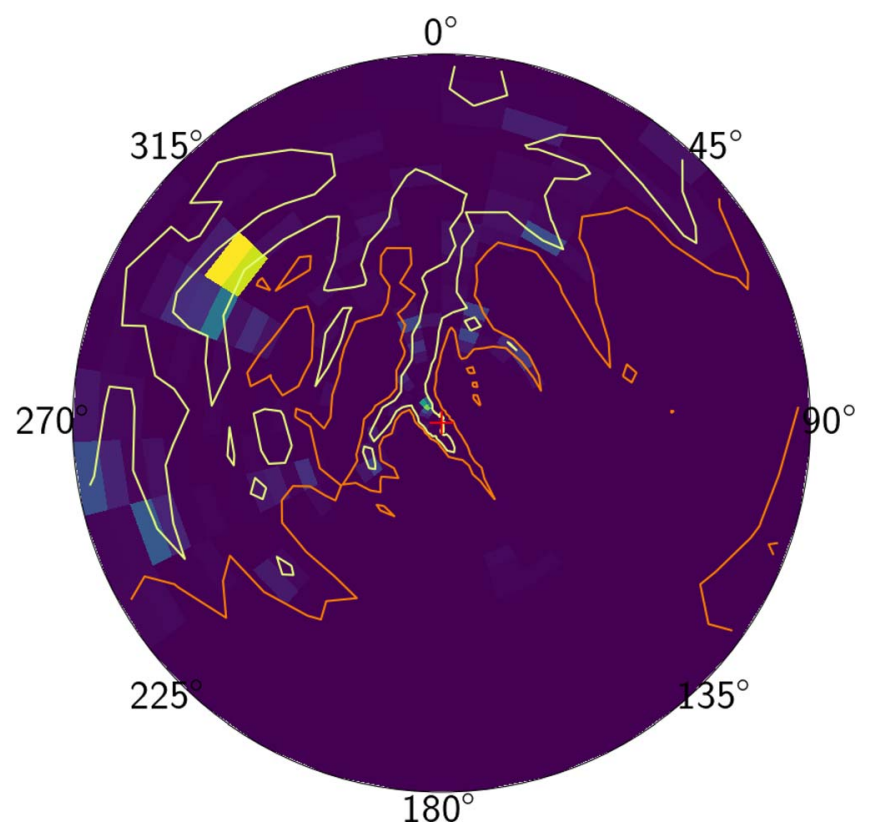

Fig. A.1. Ground map used for the model in a $100 \mathrm{~km}$ radius around the instrument (red cross). The emissions are shown in yellow (we can note the small patch of Skibotn just North-West from the instrument and the larger area covered by Troms $\varnothing$ further away). Superposed are the lines of constant altitudes at $200 \mathrm{~m}$ (yellow) and $500 \mathrm{~m}$ (orange) above sea level. These are the altitudes used for the albedo definition.

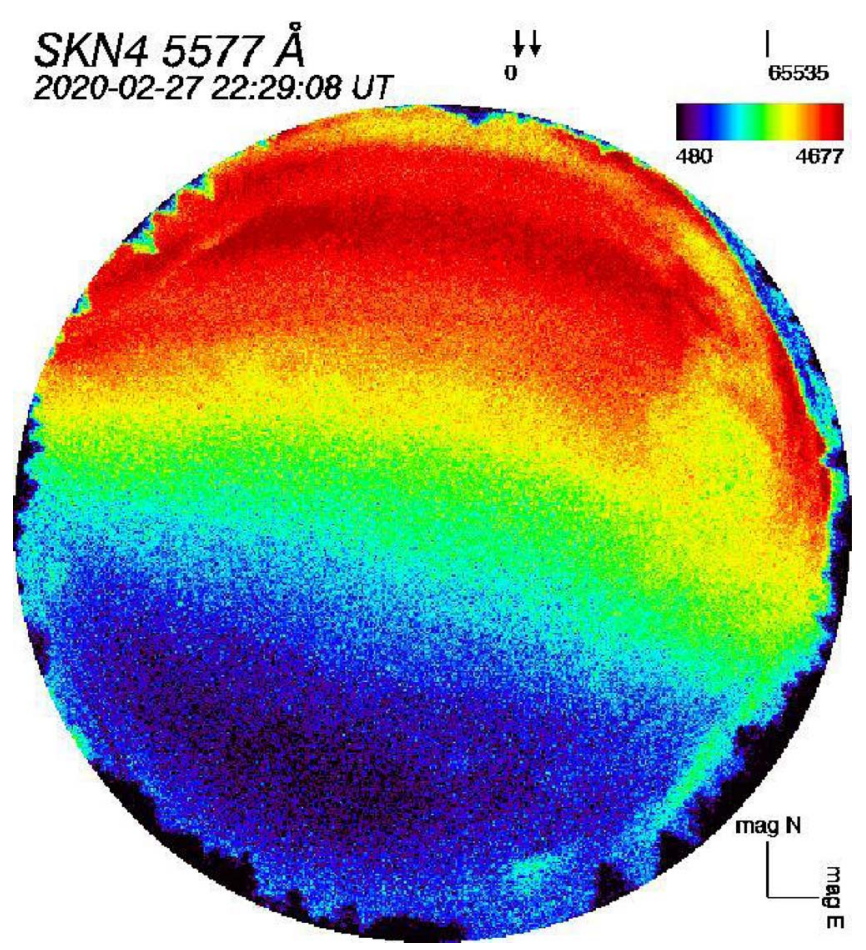

Fig. A.2. All-sky camera image of the auroral emissions in the $557.7 \mathrm{~nm}$ line used as input of the model for the observations on February 27th, 2020. The color scale gives the emission photon flux in Rayleigh unit $\left(10^{10}\right.$ photons $\left./ \mathrm{m}^{2} / \mathrm{s} / \mathrm{sr}\right)$. 


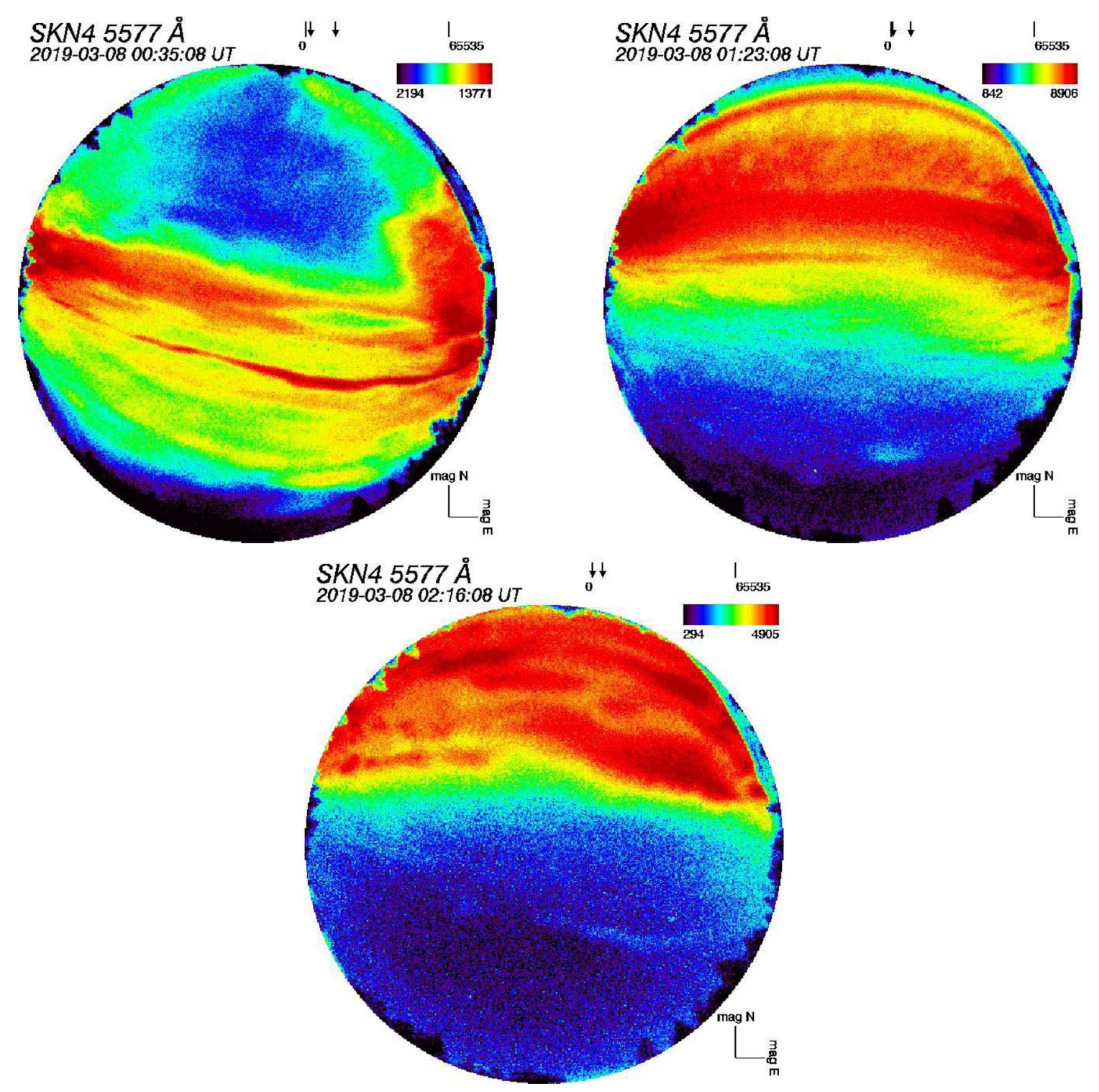

Fig. A.3. All-sky camera images of the auroral emissions in the $557.7 \mathrm{~nm}$ line used as input of the model for the purple (top left), green (top right), and blue (bottom) observations on March 8 th, 2019. The color scale gives the emission photon flux in Rayleigh units $\left(10^{10} \mathrm{photons} / \mathrm{m}^{2} / \mathrm{s} /\right.$ sr). 


\section{Appendix B: Fitting procedure}

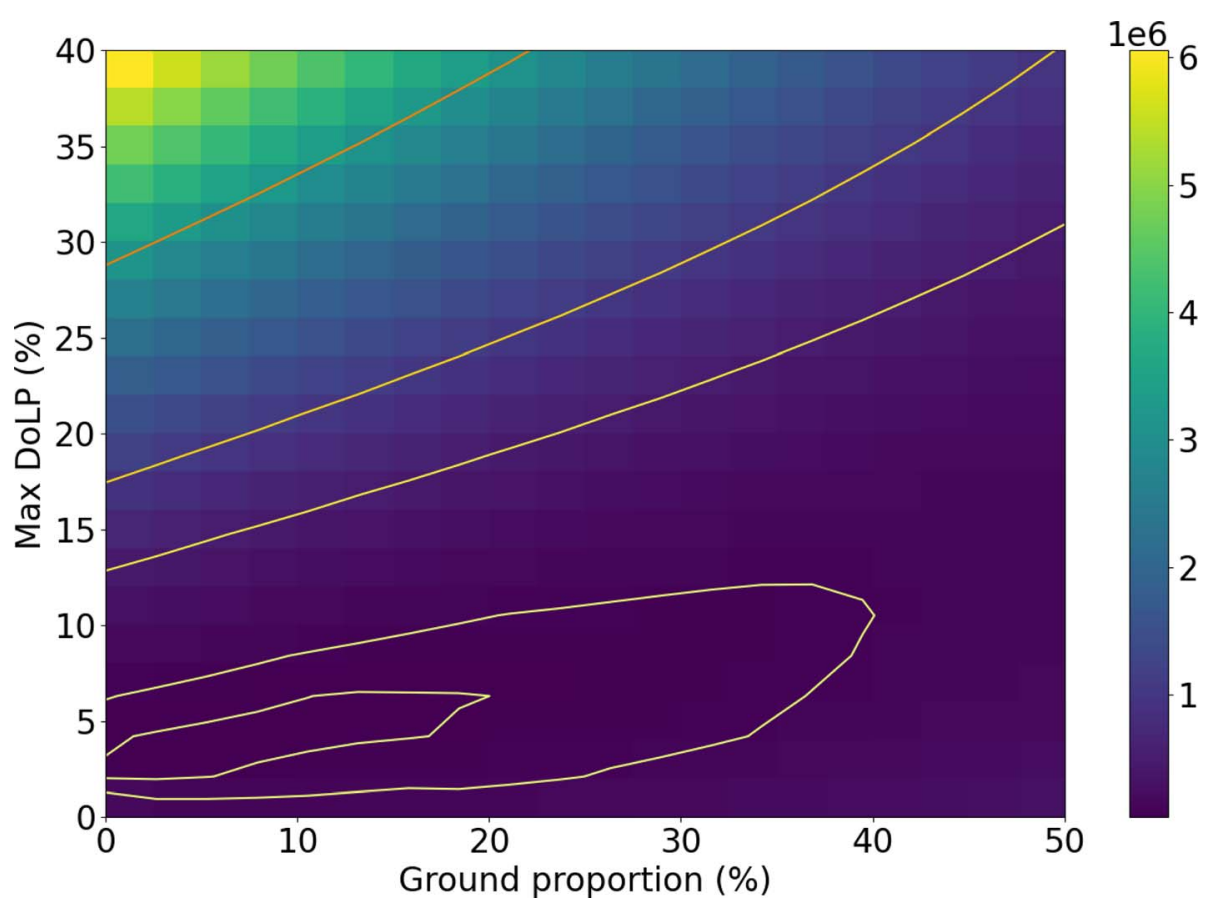

Fig. B.1. Normalized square differences between the observations (2020 green line, see Fig. 8) and the model as a function of the ground proportion and the $d_{\max }$ parameter. The azimuth is fixed at $50^{\circ}$ and the elevation at $-20^{\circ}$. The contour shows the lines of constant cost at 0.05 , $0.1,0.5,1$, and $3 \times 10^{6}$.

In order to fit the POMEROL outputs to the polarisation data (intensity, AoLP, and DoLP) we have six free parameters detailed in the following paragraphs:

- Direction (azimuth and elevation) of the electron flux (or current) exciting the neutral atmospheric targets (see Sect. 4.2).

- The maximum DoLP of the source polarisation $\left(d_{\max }\right.$, see Sect. 4.2).

- The contribution of ground emissions $\mathrm{G}$ with respect to sky source $S$, as well as the background emissions ( $K$ and $K_{\mathrm{A}}$ ) (see Sect. 2.4)

Their effects are nonlinear. Finding the optimal set of parameters is a full modeling work per se, out of the scope of this work. Still, we use the following numerical procedure.

To build a complete model taking into account all possible sources ( $G, S, K$, see Sect. 2.4), we start by adding the ground $G$ and sky $S$ contributions with a linear combination defined by a single parameter $\alpha$ as $T=\alpha G+(1-\alpha) S$. In the case where an extra background contribution is needed (i.e., purple and blue lines), this model is completed as $T_{K}=T+\gamma \mathrm{K}+\delta K_{\mathrm{A}}$, where $\gamma$ and $\delta$ are positive or negative. Note that $K_{\mathrm{A}}$ represents an auroral emission not detected on the green all-sky camera. This total model $T$ has now a direct (not scattered) auroral contribution from $S$ and $K_{\mathrm{A}}$ that can be polarised, a direct unpolarised contribution from $K$ and a scattering contribution from $G$ and $S$. To determine the polarisation of the auroral direct contribu- tion, three additional parameters are defined. The direction of the current (azimuth and elevation) and $d_{\max }$ the maximum DoLP that can be produced by the aurora. From these, we retrieve the DoLP and AoLP of the auroral direct contribution as detailed in Section 4.2.

Once this model is all set, we perform a least-square fit on the data (flux, DoLP, and AoLP normalized by their uncertainties) using the truncated Newton algorithm (Nash, 1984) (implemented in the python scipy library) using the following cost function:

$$
C=\sum_{i} \sum_{t} \frac{\left(D_{i}(t)-M_{i}(t)\right)^{2}}{\sigma_{i}^{D}(t)^{2}+\sigma_{i}^{M}(t)^{2}},
$$

where $i$ denotes the three light parameters (flux, DoLP, and AoLP), $t$ denotes the time, $D_{i}(t)$ is the observed value and $M_{i}(t)$ is the model value. $\sigma_{i}^{D}(t)$ is the error associated with the observation and $\sigma_{i}^{M}(t)$ is the error associated with the model. Relative uncertainties on the flux measurements are much smaller than those for the DoLP and AoLP. As a consequence without considering model errors, the fit is mainly driven by the flux. This causes the DoLP and AoLP to be neglected by the automatic fitting procedure, and leads to inconsistent results. However, we have seen that our forward model likely suffers from uncertainties (ground and sky maps, terrain, effect of multiple scattering, etc.) As a consequence, we introduce a modelization error on the modeled flux, considered for the sake of simplicity as $\sigma_{\text {Flux }}^{M}(t)=\frac{M_{\text {Flux }}(t)}{10}$. This way, the model fits, all three quantities (Flux, DoLP, and 


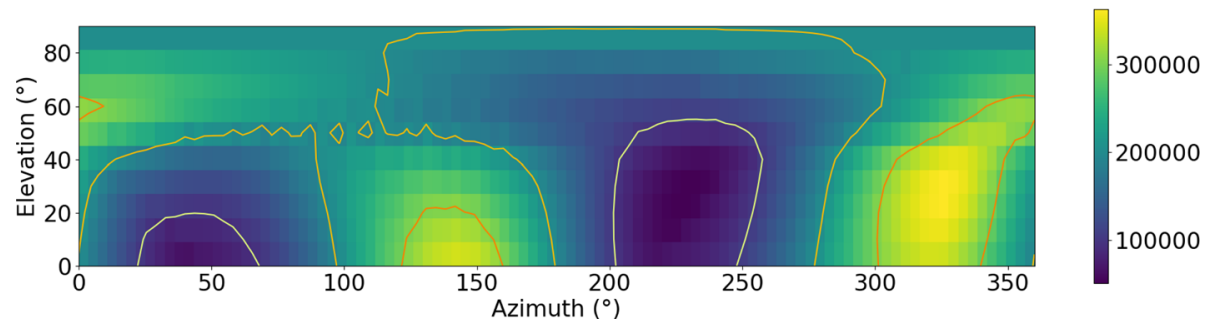

Fig. B.2. Normalized square differences between the observations (2020 green line, see Fig. 8 ) and the model as a function of the electron motion direction. The ground contribution is fixed at $20 \%$ and $d_{\max }=5 \%$. The contour shows the lines of constant cost at 1,2 , and $3 \times 10^{5}$.

AoLP) together. We do not consider any errors on the modeled DoLP and AoLP (i.e. $\sigma_{\text {DoLP }}^{M}(t)=\sigma_{\mathrm{AoLP}}^{M}(t)=0$ ), as observation errors are already significant in comparison with the signal.

In the case of the 2020 green observation, we present a detailed study of the parameter search for the best direct polarisation model. In Figure B.1, we fix the elevation at $-20^{\circ}$ and the azimuth at $50^{\circ}$ to study the relative influence of the ground contribution and $d_{\max }$. The problem is badly constrained, with many different couple of values that give similar misfit values.
In Figure B.2, we fix the ground contribution at $20 \%$ and $d_{\max }=5 \%$ to study the influence of the electron motion orientation on the fit. The azimuth varies around $360^{\circ}\left(0^{\circ}\right.$ is the North, $90^{\circ}$ the East) while the elevation is defined positive. This is sufficient since the orientation is defined modulo $180^{\circ}$ (in $3 \mathrm{D})$. We see a clear minimum around azimuth $\sim 230^{\circ}$ and elevation around $\sim 20^{\circ}$ (or azimuth $50^{\circ}$ and elevation $-20^{\circ}$ ). The orientation is better constrained than the ground contribution and the $d_{\max }$ parameters.

Cite this article as: Bosse L, Lilensten J, Gillet N, Brogniez C, Pujol O, et al. 2022. At the source of the polarisation of auroral emissions: experiments and modeling. J. Space Weather Space Clim. 12, 7. https://doi.org/10.1051/swsc/2022004. 